\title{
SINGULAR INTEGRAL EQUATIONS WITH CAUCHY KERNELS
}

BY

\author{
W. J. TRJITZINSKY
}

1. Introduction. The object of this work is to study equations of the form

$$
\begin{gathered}
\frac{1}{\pi i} \int_{L} \frac{\kappa(t, y) \phi(y) d y}{y-t}=f(t), \\
\alpha(t) \phi(t)+\frac{1}{\pi} \int_{L} \frac{\kappa(t, y) \phi(y) d y}{y-t}=f(t),
\end{gathered}
$$

where $k(t, y), \alpha(i), f(t)$ are assigned on $L$ (and satisfy hypotheses introduced in the sequel). $L$ is assumed to consist of a finite number of open arcs and closed curves; these may have only isolated points in common; except at certain points, finite in number and termed $z$-points, $L$ has a continuously turning tangent; the z-points are points at which either $L$ has two distinct tangents or several of the arcs and closed curves involved intersect. In order to give meaning to the integrals involved in (1.1), (1.2), suitable directions are to be assigned along various portions of $L$. We assume that on each open arc and on each closed curve a direction is maintained all along.

Amongst the best recent works, related to the above problem, are those of N. I. Mushelishvili( $\left.{ }^{(}\right)$, I. Vecoua $\left({ }^{2}\right), F$. Gahoff $\left({ }^{(3)}\right.$, in the sequel referred to as $(M),(V),(G)$, respectively.

In $(\mathrm{M}),(\mathrm{V}),(\mathrm{G})$ developments are given under the supposition that there are no z-points. In (G) a functional linear nonhomogeneous boundary value problem of Riemann type is studied for the case of a single simple closed curve; this boundary problem enables treatment of (1.1), (1.2) when $L$ is a single simple closed curve. The importance of $(G)$, despite the special character of the work, is due to the use of "uniformizing factors" which enable explicit solution of the problem involved. In (M) and (V) the developments are partly based on an extension of the idea in $(G)$; they utilize in part also some of the

Presented to the Society, November 24, 1944; received by the editors January 27, 1944.

(1) N. I. Mushelishvili, Application of integrals of Cauchy type to a class of singular integral equations, Travaux del'Institut mathématique de Tbilissi (Trudy Tbilisskogo matematicheskogo instituta) vol. 10 (1941) pp. 1-44; also see pp. 161-162.

(2) I. Vecoua, Integral equations with a singular kernel of Cauchy type, Travaux de l'Institut mathématique de Tbilissi (Trudy Tbilisskogo matematicheskogo instituta) vol. 10 (1941) pp. 45-72.

(3) F. Gahoff, Zur Riemannschen Randwertaufgabe, Rec. Math. (Mat. Sbornik) N.S. vol. 2 (1937) pp. 673-683. 
early ideas of Carleman; to a considerable extent (M) and (V) involve new ideas. In (M), $L$ consists of a finite number of open arcs without common points; in (V), $L$ is a finite number of simple closed curves without common points. As remarked in (M), a synthesis of (M), (V) would not be difficult, of course if no $z$-points were involved. Inasmuch as we allow presence of $z$-points, we encounter substantial new difficulties.

In view of the stated purpose a study of integrals of the form

$$
\Phi(x)=\int_{L} \frac{F(y) d y}{y-x}
$$

where $|F(y)|$ is integrable along $L$ and $F(y)$ is of a Hölder class on $L$ (except, perhaps, at end points of $L$ and at $z$-points), is essential. In particular we shall need to know about existence (as well as the values of the limits obtained when $x$ tends to a point $t$ on $L$ either within $C(L)$ (the open set complementary to $L$ ) or along $L$. For $t \neq z$ the situation is, of course, well known(4). In \$2 we extend Plemelj's formulas to z-points. In this connection we shall also need to extend to z-points the notion of Cauchy principal values (cf. §2).

Use will be made of the following notation. Generically $c^{*}$ will denote any positive constant; end points of $L$ will be designated by $c ; x$ will be in $C(L)$ (unless stated otherwise); $t, y, \tau$ will be on $L$, distinct from $z, c$-values (unless the contrary is specified). $H_{h}$ will designate a Hölder class with exponent $h$ $(0<h \leqq 1)$.

In $\$ 3$ a study is given of continuity properties of Cauchy principal values in the vicinity of $z$-points. The main result of $\$ 4$ is solution of the nonhomogeneous Riemann type boundary value problem (4.19); this section is vital for the subsequent pages. In $\$ 5$ we establish Theorem 5.1, giving the general solution of the problem (5.1) and Theorem 5.2, which relates to equation (1.1). The main result of $\$ 6$ is embodied in Theorem 6.2 and refurs to equation (1.2). The essential point with respect to Theorems 5.2, 6.2 is that the given integral equations (1.1), (1.2) (under stated conditions) are shown to be equivalent to regular Fredholm integral equations of the second kind. All these solutions are obtained on $L$, excluding $z, c$-points. In $\$ 7$ (Theorem 7.1) it is shown that at some of the $z$-points the solutions of (1.1), (1.2) satisfy certain modified integral equations (7.2), (7.6).

2. Integrals (1.3). Let $z$ be a particular z-point. In a sufficiently small neighborhood of $z$ the portion $L_{0}$ of $L$ will consist of curves $C_{\nu}(\nu=1, \cdots, m)$ such that the following holds. $C_{\nu}$ extends from $z$ to a point $z+A_{\nu}$; the $C_{\nu}$ have no points in common, except $z$. For $y$ on $C_{\nu}$ one has

$$
y=z+t e^{i v_{\nu}},
$$$$
y_{\nu}^{\prime}=\alpha_{\nu}+\theta_{\nu}(t)
$$$$
\left(0<t \leqq\left|A_{\nu}\right|\right),
$$

(') See J. Plemelj, Ein Ergänzungssatz zur Cauchyschen Integraldarstellung Analytischer Funktionen, Randwerte betreffend, Monatshefte für Mathematik und Physik vol. 19 (1908) pp. 205-210. 
where $\theta_{\nu}(t)$ is continuous and

$$
\lim \theta_{\nu}(t)=0
$$$$
\text { (as } y \rightarrow z) \text {. }
$$

Clearly $\alpha_{v}$ is the limiting direction of $C$, at $z$. The $C_{v}$ will be supposed to be ordered in the counterclockwise direction. Thus

$$
0<\alpha_{1} \leqq \alpha_{2} \leqq \cdots \leqq \alpha_{m} \leqq 2 \pi ; \quad \alpha_{m+1}=\alpha_{1}+2 \pi .
$$

We shall deal in this section with z-points for which no two of the $\alpha_{j}$ are equal. There is no loss of generality if one takes $\left|A_{\nu}\right|=A(\nu=1, \cdots, m)$. We let $R$, denote the part of the circular region $|y-z| \leqq A$ extending from $C_{\nu}$ to $C_{v+1}(\nu=1, \cdots, m) ; \alpha_{1}=\alpha_{m+1}, C_{1}=C_{m+1}, A_{1}=A_{m+1}$.

Since our intention now is to extend the notion of Cauchy principal values we shall have to assume that integrations along the $C_{\nu}$ are not all directed towards $z$ and are neither all directed away from $z$. Let integrations be towards $z$ along the curves $C_{i_{1}}, C_{i_{2}}, \cdots, C_{i_{k}}$ and away from $z$ along $C_{e_{1}}, C_{e_{2}}, \cdots, C_{\theta_{m-k}}$, where

$$
i_{1}<i_{2}<\cdots<i_{k} ; e_{1}<e_{2}<\cdots<e_{m-\kappa} ; 1 \leqq \kappa<m .
$$

Let

$$
a_{\nu}=z+\sigma_{\nu} e^{i \delta_{\nu},} \quad \delta_{\nu}=\alpha_{\nu}+\theta_{\nu}\left(\sigma_{\nu}\right)
$$

be a point on $C_{v}$. Designate by $L^{\prime}$ the part of $L_{0}$ consisting of the portions of the $C_{\nu}$ extending from $z$ to $a_{\nu}(\nu=1, \cdots, m)$; let $L^{\prime \prime}=L_{0}-L^{\prime}$. On writing $\alpha=z+A_{i j}, \delta=a_{i j}, \beta=a_{e_{k}}, \gamma=z+A_{e_{k}}$ one has

$$
\begin{aligned}
\int_{L^{\prime \prime}} \frac{d y}{y-z}= & \sum_{j} \int_{\alpha}^{\delta} \frac{d y}{y-z}+\sum_{\alpha} \int_{\beta}^{\gamma} \frac{d y}{y-z} \\
= & \sum_{\alpha} \log A_{e_{\alpha}}-\sum_{j} \log A_{i j}+\sum_{j} \log \left[\sigma_{i j} \exp i \delta_{i j}\right] \\
& -\sum_{\alpha} \log \left[\sigma_{e_{\alpha}} \exp i \delta_{e_{k}}\right] .
\end{aligned}
$$

The two sums last displayed combine into the logarithth of

$$
\frac{\sigma_{i_{1}} \sigma_{i_{2}} \cdots}{\sigma_{\theta_{1}} \sigma_{\theta_{2}} \cdots} \exp i\left[\left(\delta_{i_{1}}+\delta_{i_{2}}+\cdots\right)-\left(\delta_{\theta_{1}}+\delta_{\theta_{2}}+\cdots\right)\right] \text {. }
$$

We take

$$
0<\epsilon<1 ; \quad \sigma_{\nu}=\epsilon^{\tau_{\nu}} ; \quad 1>\tau_{\nu}>0
$$$$
(\nu=1, \cdots, m)
$$

and choose the $\tau_{y}$ so that

$$
\tau_{i_{1}}+\tau_{i_{2}}+\cdots+\tau_{i_{k}}=\tau_{e_{1}}+\tau_{e_{2}}+\cdots+\tau_{e_{m-k}} ;
$$

at the same time $\epsilon$ will be taken certainly so small that $\sigma_{\nu}<A(\nu=1, \cdots, m)$. The absolute value of (2.4) will then be equal to unity. In consequence of (2.3) and (2.1a) the expression (2.4) is seen to tend to 


$$
\exp i\left[\left(\alpha_{i_{1}}+\alpha_{i_{2}}+\cdots\right)-\left(\alpha_{e_{1}}+\alpha_{e_{2}}+\cdots\right)\right],
$$

as $\epsilon \rightarrow 0$. Thus

$$
\begin{gathered}
\lim _{\epsilon \rightarrow 0} \int_{L^{\prime \prime}} \frac{d y}{y-z}=\log \frac{A_{e_{1}} A_{e_{2}} \cdots}{A_{i_{1}} A_{i_{2}} \cdots}+i \Lambda, \\
\Lambda=\left(\alpha_{i_{1}}+\cdots+\alpha_{i_{k}}\right)-\left(\alpha_{\theta_{1}}+\cdots+\alpha_{\theta_{m-k}}\right) .
\end{gathered}
$$

We observe that when we choose $\sigma_{\nu}$ as in (2.4a) and let $\epsilon \rightarrow 0$ the set $L^{\prime \prime}$ tends to $L_{0}$.

With (1.3) in view we let

$$
\Phi_{0}(x)=\int_{L_{0}} \frac{F(y) d y}{y-x}
$$

and note the equality

$$
\int_{L^{\prime \prime}} \frac{F(y) d y}{y-z}=F(z) \int_{L^{\prime \prime}} \frac{d y}{y-z}+\int_{L^{\prime \prime}} \frac{F(y)-F(z)}{y-z} d y .
$$

If $s$ denotes arc length measured from $z$ along $C_{v}$, then

$$
|d y|=d s, \quad \lim _{s \rightarrow 0} \frac{d t}{d s}=1 .
$$

Hence

$$
|d y|<c^{*} d t
$$

along $C_{\nu}$ if $A$ is taken small enough, and, since $F$ is of a Hölder class, say $H_{h}$,

$$
\left|\frac{F(y)-F(z)}{y-z} d y\right|<c^{*} t^{h-1} d t \quad\left(y \text { on } C_{r} ; 0<t \leqq A\right) .
$$

The integral of the second member, extended over $0 \leqq t \leqq A$, exists; whence

$$
\lim _{a \rightarrow 0} \int_{L^{\prime \prime}} \frac{F(y)-F(z)}{y-z} d y=\int_{L_{0}} \frac{F(y)-F(z)}{y-z} d y .
$$

The integral last displayed is in the ordinary sense. By (2.5a), (2.4c) and the above

$$
\begin{aligned}
\lim _{a \rightarrow 0} \int_{L^{\prime \prime}} \frac{F(y) d y}{y-z}= & F(z)\left[\log \frac{A_{e_{1}} A_{e_{2}} \cdots}{A_{i_{1}} A_{i_{2}} \cdots}+i \Lambda\right] \\
& +\int_{L_{0}} \frac{F(y)-F(z)}{y-z} d y
\end{aligned}
$$

(cf. (2.4d)). We are now ready to introduce the following definition. 
Definition 2.1. Suppose $F \subset H_{h}(0<h \leqq 1)$ on $L$ in the vicinity of $z$ and $|F|$ is integrable over $L$. As a matter of definition we shall write

$$
\int_{L} \frac{F(y) d y}{y-z}=\int_{L-L_{0}} \frac{F(y) d y}{y-z}+\int_{L_{0}} \frac{F(y) d y}{y-z},
$$

where

$$
\int_{L_{0}} \frac{F(y) d y}{y-z}=\lim _{a \rightarrow 0} \int_{L^{\prime \prime}} \frac{F(y) d y}{y-z}=\text { second member in (2.7), }
$$

$L^{\prime \prime}$ being defined as above (cf. (2.4a), (2.4b)). The first members in (2.7a), (2.7b) will be termed "Cauchy principal values." The above definition is justified since the second member in (2.7a) is independent of the $\tau_{v}$ and since Cauchy principal values in the ordinary sense present a special case of the situation in. volved in the definition.

Before we study limits of an integral (1.3) at $z$ we shall observe that, with $\omega_{i}(j=1, \cdots, m)$ constituting a set of distinct, possibly complex-valued, numbers all different from zero, one has

$$
\begin{aligned}
\frac{1}{u-\omega_{j}} & =\sum_{s=1}^{m} \lambda_{j, 8} \frac{u^{m-s}}{\left(u-\omega_{1}\right) \cdots\left(u-\omega_{m}\right)} & (j=1, \cdots, m), \\
\lambda_{j, 8} & =(-1)^{s-1} \sum^{(j)} \omega_{p_{1}} \cdots \omega_{p_{s-1}}, & \lambda_{j, 1}=1,
\end{aligned}
$$

where the summation is over values $p_{1}, \cdots, p_{s-1}$ ranging over $1,2, \cdots$, $j-1, j+1, \cdots, m$, subject to inequalities $p_{1}<\cdots<p_{s-1}$. We write

$$
\Phi_{0}(x)=\int_{L_{0}} \frac{F(y) d y}{y-x}=F(z) \int_{L_{0}} \frac{d y}{y-x}+\int_{L_{0}} \frac{F(y)-F(z)}{y-x} d y
$$

and, on taking account of (2.8), obtain

$$
\begin{aligned}
& \Phi_{0}\left(z+\omega_{j} \epsilon\right)=F(z) \int_{L_{0}} \frac{d y}{u-\omega_{j} \epsilon}+\sum_{s=1}^{m} \lambda_{j, s} \Gamma_{s}, \\
& \Gamma_{s}=\int_{L_{0}} \frac{\epsilon^{s-1} u^{m-s}[F(y)-F(z)] d y}{\left(u-\omega_{1} \epsilon\right) \cdots\left(u-\omega_{m} \epsilon\right)} \quad(j, s=1, \cdots, m) ;
\end{aligned}
$$

here and in the sequel $u=y-z$; the $\lambda_{j, 8}$ are defined by (2.8a). Henceforth let $m=2 \mu, \kappa=\mu$; one thus may take $\sigma_{\nu}=\sigma=\epsilon^{\tau}(0<\tau<1)$.

We define regions

$$
W_{\nu}(\xi)=\left\{\alpha_{\nu}+\xi \leqq \arg (y-z) \leqq \alpha_{\nu+1}-\xi ;|y-z| \leqq A\right\}
$$

$(\xi>0 ; \nu=1, \cdots, 2 \mu)$. As is possible, $A$ is to be chosen, depending on $\xi$, sufficiently small so that $W_{\nu}(\xi)$ is a subset of the region $R_{\nu}$, defined subsequent to $(2.1 \mathrm{~b})$. The $\omega_{j}$ in $(2.9 \mathrm{a}),(2.9 \mathrm{~b})$ we shall take so that $z+\omega_{j} \in$ is in $W_{j}(\xi)$. Thus 


$$
\omega_{j}=e^{i \omega_{j}^{\prime}}, \quad \alpha_{j}+\xi \leqq \omega_{j}^{\prime} \leqq \alpha_{j+1}-\xi ;
$$

then

$$
\left|\omega_{j}^{\prime}-\alpha_{\bullet}\right| \geqq \xi \text {. }
$$

Recalling the definitions of $L^{\prime}, L^{\prime \prime}$ we write $\Gamma_{\imath}=\Gamma !+\Gamma_{\iota}^{\prime \prime}$, where

$$
\begin{gathered}
\Gamma_{\bullet}^{\prime}=\int_{L^{\prime}} \frac{\epsilon^{s-1} u^{m-s}[F(y)-F(z)] d y}{\left(u-\omega_{1} \epsilon\right) \cdots\left(u-\omega_{m} \epsilon\right)}, \\
\Gamma_{\bullet}^{\prime \prime}=\int_{L^{\prime \prime}} \frac{\epsilon^{\prime-1} u^{m-s}[F(y)-F(z)] d y}{\left(u-\omega_{1} \epsilon\right) \cdots\left(u-\omega_{m} \epsilon\right)} .
\end{gathered}
$$

Let $S_{\nu}$ be the arc of a circle of center $z$ extending from $z+A_{i,}$ to $z+A_{e_{p}}$; of the two possible arcs of this description $S_{v}$ is to be the one along which one can pass in the counterclockwise direction from end point to end point, beginning from that one of the end points $z+A_{0}$ for which the angle of $A_{0}$ is less than the corresponding angle for the other end point. The interior region bounded by the simple closed curve $K_{\nu}=S_{\nu}+C_{i_{y}}+C_{\theta_{\nu}}$ will be designated by $\Delta_{v}$. Along $C_{i,}, C_{0,}$ as indicated previously, directions are assigned towards and away from $z$, respectively; along $S_{\nu}$ we assign a direction so that $K$, has an assigned direction (all along). Thus, if $\alpha_{i_{\nu}}<\alpha_{\varepsilon_{v}}$, integration in

$$
\int_{K} \frac{d y}{u-\omega_{j \epsilon}}
$$

will be extended in the negative direction (leaving $\Delta$, on the right); integration in (2.12) will be in the positive direction if $\alpha_{i,}>\alpha_{\theta_{v}}$. Consequently (2.12) has values $-2 \pi i$ (if $\alpha_{i_{y}}<\alpha_{\theta_{y}}$ ) and $2 \pi i$ (if $\alpha_{i_{y}}>\alpha_{e_{y}}$ ), whenever $z+\omega_{j} \epsilon$ is in $\Delta_{y}$; (2.12) has value zero if $z+\omega_{j} \epsilon$ is exterior $\Delta_{y}$. Whence

$$
\int_{K_{\nu}} \frac{d y}{u-\omega_{j \epsilon}}=\left(\int_{C_{i,}}+\int_{c_{\sigma_{\nu}}}\right) \frac{d y}{u-\omega_{j \epsilon}}+\int_{s_{\nu}} \frac{d y}{u-\omega_{j \epsilon}}= \pm 2 \pi i
$$

$\left(z+\omega_{j} \epsilon\right.$ in $\Delta_{v}$; sign of $\left.\alpha_{i_{\nu}}-\alpha_{\theta_{\nu}}\right)$. Integration along $S_{\nu}$ will be from $z+A_{e,}$ to $z+A_{i,}$ in any case. Thus

$$
\left(\int_{C_{i,}}+\int_{C_{\theta_{\nu}}}\right) \frac{d y}{u-\omega_{j \epsilon}}= \pm 2 \pi i-\int_{s_{k}} \frac{d y}{u-\omega_{j \epsilon}}= \pm 2 \pi i+\log \frac{A_{\theta_{\nu}}-\omega_{j \epsilon}}{A_{i_{\nu}}-\omega_{j \epsilon}}
$$

if $z+\omega_{j} \epsilon$ is in $\Delta_{v}$; in the case when $z+\omega_{j} \in$ is exterior $\Delta_{\nu}$ we replace $\pm 2 \pi i$, above, by zero, Hence

$$
\lim _{a \rightarrow 0}\left(\int_{C_{i_{p}}}+\int_{C_{e_{\nu}}}\right) \frac{d y}{u-\omega_{j} \epsilon}=\log \frac{A_{e_{v}}}{A_{i_{p}}}+2 \pi i \delta_{v, j},
$$

where 


$$
\delta_{v, j}=\left\{\begin{array}{r}
1 \\
-1 \\
0
\end{array}\right.
$$

$$
\begin{aligned}
& \text { (if } \alpha_{i_{\nu}}-\alpha_{\theta_{\nu}}>0 ; z+\omega_{j \in} \text { in } \Delta_{v} \text { ); } \\
& \text { (if } \alpha_{i_{\nu}}-\alpha_{\theta_{\nu}}<0 ; z+\omega_{j \in} \text { in } \Delta_{v} \text { ); } \\
& \left(z+\omega_{j} \in \text { exterior } \Delta_{v}\right) \text {. }
\end{aligned}
$$

The region $W_{j}(\xi)$ either lies in $\Delta_{\nu}$ or lies exterior $\Delta_{\nu}$; thus $\delta_{\nu, j}$ has a fixed value depending on $\nu$ and $j$ only. In view of (2.13)

$$
\lim \int_{L_{0}} \frac{d y}{u-\omega_{j \epsilon}}=\log \frac{A_{\theta_{1}} \cdots A_{o_{\mu}}}{A_{i_{1}} \cdots A_{i_{\mu}}}+2 \pi i \Lambda\left(\omega_{j}\right),
$$

where

$$
\Lambda\left(\omega_{j}\right)=\sum_{\nu=1}^{\mu} \delta_{\nu, j}
$$

(cf. (2.13a)). By (2.4c) and (2.14)

$$
\lim \int_{L_{0}} \frac{d y}{u-\omega_{j} \epsilon}=\int_{L_{0}} \frac{d y}{y-z}+2 \pi i \Lambda\left(\omega_{j}\right)-i \Lambda .
$$

We recall $(2.10 \mathrm{~b})$ and take $A$, if necessary, suitably small so that

$$
\left|\omega_{\nu}^{\prime}-y_{j}^{\prime}(t)\right| \geqq q>0,
$$

where $q \leqq \xi$ and $q$ is independent of $t$. Now

$$
\left|u-\omega_{\nu} \epsilon\right|=\left|t \exp \left(i y_{j}^{\prime}(t)\right)-\epsilon \exp \left(i \omega_{\nu}^{\prime}\right)\right|=\left|t-\epsilon \exp \left(i\left(\omega_{\nu}^{\prime}-y_{j}^{\prime}\right)\right)\right|
$$

for $y$ on $C_{j}$. In view of (2.15) the point represented in the complex $\lambda$-plane by $r_{\nu, j}=\epsilon \exp \left(i\left(\omega_{\nu}^{\prime}-y_{j}^{\prime}\right)\right)$ is on the circular arc $|\lambda|=\epsilon, q \leqq$ angle $\lambda \leqq 2 \pi-q$, while $t$ is a point of the interval $0 \leqq t \leqq A$. We observe that $\left|t-r_{p, j}\right|$ is not less than the distance from $t$ to an end point of the above arc. Thus $\left|u-\omega_{\nu} \epsilon\right|=\left|t-r_{\nu, j}\right|$ $\geqq\left[t^{2}-2 t \epsilon \cos q+\epsilon^{2}\right]^{1 / 2}$ for $y$ on $C_{j}$. With $t$ fixed, the function of $\epsilon$, $t^{2}-2 t \epsilon \cos q+\epsilon^{2}$, assumes its lower bound for $\epsilon=t \cos q$; this bound is positive; thus

$$
\left|u-\omega_{\nu} \epsilon\right| \geqq t|\sin q|
$$

$\left(y\right.$ on $\left.C_{j}\right)$;

by symmetry of [..] in $t, \epsilon$ we also obtain

$$
\left|u-\omega_{\nu} \epsilon\right| \geqq \epsilon|\sin q|
$$

( $y$ on $C_{j}$ );

these inequalities hold for $\nu, j=1, \cdots, \mu$. Applying (2.16a) to $s-1$ factors and (2.16) to the remaining factors we obtain

$$
\left|u-\omega_{1} \epsilon\right| \cdots\left|u-\omega_{m} \epsilon\right| \geqq\left|\sin ^{m} q\right| \epsilon^{s-1} t^{m-s+1}
$$

for $y$ on $C_{1}, \cdots, C_{m}$-thus for $y$ on $L_{0}$. Whence by (2.6) and (2.11)

$$
\left|\Gamma_{\bullet}^{\prime}\right|<c^{*} \int_{L^{\prime}} \frac{\epsilon^{s-1} t^{m-s+h} d t}{\left|u-\omega_{1} \epsilon\right| \cdots\left|u-\omega_{m} \epsilon\right|}<c^{*} \sigma^{h}
$$


where $\sigma=\epsilon^{\tau}(0<\tau<1)$. Accordingly

$$
\lim \Gamma_{s}^{\prime}=0
$$$$
(s=1, \cdots, m)
$$

Since $\sigma>\left|\omega_{\nu} \epsilon\right|=\epsilon$, one has

$$
\left|u-\omega_{\nu} \epsilon\right| \geqq \sigma-\epsilon, \quad\left|u-\omega_{\nu} \epsilon\right|^{-1}<c^{*} \epsilon^{-r}
$$

for $y$ in $L^{\prime \prime}$. Hence, by (2.11), $\left|\Gamma_{s}^{\prime \prime}\right|$ is bounded by $c^{*} \epsilon^{\theta-1-m r}$. Thus

$$
\lim \Gamma_{\bullet}^{\prime \prime}=0
$$$$
(s=2, \cdots, m) \text {, }
$$

provided $\tau$ is defined so that $0<\tau<1 / m$.

The integral $\Gamma_{1}^{\prime \prime}$ requires special investigation. We write

$$
W_{\epsilon}=\Gamma_{1}^{\prime \prime}=\int_{L^{\prime \prime}} \frac{u^{m-1}[F(y)-F(z)] d y}{\left(u-\omega_{1} \epsilon\right) \cdots\left(u-\omega_{m} \epsilon\right)}
$$

and form the difference

$$
W_{\epsilon}-W_{0}=\epsilon \int_{L^{\prime \prime}} \frac{N(u, \epsilon)[F(y)-F(z)] d y}{\left(u-\omega_{1} \epsilon\right) \cdots\left(u-\omega_{m} \epsilon\right) u}
$$

and note that on $L_{0},|N|<\kappa^{\prime}$, where $\kappa^{\prime}$ is independent of $\omega_{1}, \cdots, \omega_{m}, \epsilon, u$. Using the fact that $F(y) \subset H_{h}$ and the inequalities after (2.17), we infer that $\left|W_{\mathrm{c}}-W_{0}\right|$ is bounded by $c^{*} \epsilon^{1-m r}$. Hence $W_{\mathrm{c}}-W_{0} \rightarrow 0$ with $\epsilon_{\text {. Now }}$

$$
\lim _{a \rightarrow 0} W_{0}=\lim _{\leftrightarrow \rightarrow 0} \int_{L^{\prime \prime}} \frac{F(y)-F(z)}{y-z} d y=\int_{L_{0}} \frac{F(y)-F(z)}{y-z} d y
$$

exists since $F(y) \subset H_{h}$. Accordingly

$$
\lim W_{\epsilon}=\lim \Gamma_{1}^{\prime \prime}=\int_{L_{0}} \frac{F(y)-F(z)}{y-z} d y .
$$

By virtue of (2.9a), (2.14b), (2.11), (2.17), $\Phi_{0}\left(z+\omega_{j} \epsilon\right)$ tends to

$$
F(z)\left\{-i \Lambda+i \Lambda\left(\omega_{j}\right) 2 \pi+\int_{L_{0}} \frac{d y}{y-z}\right\}+\sum_{s} \lambda_{j, 8} \lim \Gamma_{\bullet}^{\prime \prime} .
$$

Hence by (2.18), (2.19a), $\Phi_{0}\left(z+\omega_{j} \epsilon\right)$ tends to

$$
F(z)\left\{-i \Lambda+i \Lambda\left(\omega_{j}\right) 2 \pi+\int_{L_{0}} \frac{d y}{y-z}\right\}+\lambda_{j, 1} \int_{L_{0}} \frac{F(y)-F(z)}{y-z} d y .
$$

On taking account of (2.8a) it is deduced that

$$
\begin{aligned}
& \Phi_{0}^{. j}(z)=\lim _{e} \Phi_{0}\left(z+\omega_{j \epsilon}\right)=\int_{L_{0}} \frac{F(y) d y}{y-z}+i \Omega\left(\omega_{j}\right) F(z), \\
& \Omega\left(\omega_{j}\right)=-\Lambda+2 \pi \Lambda\left(\omega_{j}\right)
\end{aligned}
$$


for $z+\omega, \epsilon$ in $W_{j}(\xi)$. This is the desired extension of Plemelj's formulas. The formula remains valid when $L_{0}$ is replaced by $L$.

The quantity $\Lambda\left(\omega_{j}\right)$ has been defined on the basis of the specific labelling of the various $C_{i,}$ and $C_{\theta,}$ curves given by (2.2). However, the formula (2.20). for the combination $\Omega\left(\omega_{j}\right)$ and the definition (2.4d) of $\Lambda$ (with $m=2 \mu, \kappa=\mu$ ) show that these two quantities are independent of the labelling of the curves. Accordingly this is also true of $\Lambda\left(\omega_{j}\right)$. We make use of this fact in the discussion below.

Consider the important special case when, in the neighborhood of $z, L$ consists of $\mu(>1)$ curves, each extending in two directions from $z$ and each having at $z$ a unique tangent. We thus have

$$
\begin{gathered}
\theta<\alpha_{1}<\cdots<\alpha_{\mu} \leqq \pi, \quad \alpha_{\mu+\nu}=\alpha_{\nu}+\pi \quad(\nu=1, \cdots, \mu), \\
\alpha_{2 \mu+1}=\alpha_{1}+2 \pi .
\end{gathered}
$$

In this case, we name the curves $C_{\theta_{v}}$ and $C_{i}$, so that the (unordered) pair $\left(C_{\theta_{\nu}}, C_{i_{\nu}}\right)$ is identical with the pair $\left(C_{\nu}, C_{\mu+i}\right)$. One will then have

$$
\Lambda\left(\omega_{j}\right)=\sum_{\nu=1}^{j} \epsilon_{\nu}, \quad \Lambda\left(\omega_{\mu+j}\right)=\sum_{\nu=j+1}^{\mu} \epsilon_{\nu}
$$

for $j=1, \cdots, \mu$, the $\epsilon_{\nu}$ being defined by

$$
\epsilon_{\nu}=\left\{\begin{aligned}
1 & \left(\text { if } C_{\nu} \text { is a } C_{o s}\right. \text { curve) } \\
-1 & \text { (if } C_{\nu} \text { is a } C_{i,} \text { curve) } .
\end{aligned}\right.
$$

Also

$$
\Lambda=\sum_{s=1}^{\mu}\left(\alpha_{i_{s}}-\alpha_{\theta_{s}}\right)=\sum_{\nu=1}^{\mu} \epsilon_{\nu}\left(\alpha_{\nu+\mu}-\alpha_{v}\right)=\pi \sum_{v=1}^{\mu} \epsilon_{\nu}
$$

Whence in the special case under consideration we have

$$
\Omega\left(\omega_{j}\right)=\pi\left[-\sum_{1}^{\mu} \epsilon_{\nu}+2 \sum_{1}^{j} \epsilon_{\nu}\right]=-\Omega\left(\omega_{\mu+j}\right) \quad(j=1, \cdots, \mu) .
$$

In the case considered by Plemelj one may put $\mu=1$ and $0<\alpha_{1} \leqq \pi$, $\alpha_{2}=\alpha_{1}+\pi ; \epsilon_{1}$ of (2.21a) will be -1 if $C_{1}$ is directed towards $z$ and 1 in the contrary case; one then has $\Omega\left(\omega_{1}\right)=\pi \epsilon_{1}, \Omega\left(\omega_{2}\right)=-\pi \epsilon_{1}$ and (2.20) becomes

$$
\begin{aligned}
& \lim _{\cdot} \frac{1}{2 \pi i} \Phi_{0}\left(z+\omega_{1} \epsilon\right)= \frac{1}{2 \pi i} \Phi_{0}(z)+\frac{\epsilon_{1}}{2} F(z) \\
&\left(\alpha_{1}+\xi \leqq \omega_{1}^{\prime} \leqq \alpha_{1}+\pi-\xi\right), \\
& \lim _{\cdot} \frac{1}{2 \pi i} \Phi_{0}\left(z+\omega_{2} \epsilon\right)= \frac{1}{2 \pi i} \Phi_{0}(z)-\frac{\epsilon_{1}}{2} F(z) \\
&\left(\alpha_{1}+\pi+\xi \leqq \omega_{2}^{\prime} \leqq \alpha_{1}+2 \pi-\xi\right),
\end{aligned}
$$


where $\Phi_{0}(z)$ is a Cauchy principal value and $\xi(>0)$ is arbitrarily small. If we face in the direction of $C_{1}+C_{2}$ and call the left side + and the right side (as is done in (M)), then considering (2.22) for $C_{1}$ directed towards $z$ (when $\epsilon_{1}=-1$ ) and in the contrary case (when $\epsilon_{2}=1$ ) we obtain

$$
\begin{aligned}
& \frac{1}{2 \pi i} \Phi_{0}^{+}(z)=\frac{1}{2 \pi i} \Phi_{0}(z)+\frac{1}{2} F(z), \\
& \frac{1}{2 \pi i} \Phi_{0}^{-}(z)=\frac{1}{2 \pi i} \Phi_{0}(z)-\frac{1}{2} F(z),
\end{aligned}
$$

here $\Phi_{0}+(z)\left[\Phi_{0}-(z)\right]$ is limit of $\Phi_{0}(z+\omega \epsilon)$ as $z+\omega \epsilon$ tends to $z$ from the positive [negative] side; these are precisely the original Plemelj formulas.

Another special case (not included in the Plemelj formulas) is the one in which $\mu=1$ and

$$
0<\alpha_{1}<\alpha_{2} \leqq 2 \pi ; \quad \alpha_{2}-\alpha_{1} \neq \pi .
$$

To fix ideas suppose the direction along $C_{1}$ is away from $z$. Thus $L_{0}$ will consist of the open arc $C_{1}+C_{2}$ with a known direction along it. At $z$ this arc will have two tangents, making angles $\alpha_{1}, \alpha_{2}$. We write $\alpha_{1}+\xi \leqq \omega_{1}^{\prime} \leqq \alpha_{2}-\xi$, $\alpha_{2}+\xi \leqq \omega_{2}^{\prime} \leqq \alpha_{1}+2 \pi-\xi$. Now $e_{1}=1, i_{1}=2$; moreover, $\Delta_{1}$ introduced subsequent to $(2.11 \mathrm{a})$ is the region

$$
\alpha_{1} \leqq \arg (y-z) \leqq \alpha_{2} ; \quad|y-z| \leqq A ;
$$

thus $W_{1}(\xi)$ is a subset of $\Delta_{1}$ and $W_{2}(\xi)$ lies exterior $\Delta_{1}$; also

$$
\alpha_{i 1}-\alpha_{e 1}=\alpha_{2}-\alpha_{1}>0 \text {. }
$$

Whence in view of $(2.13 a)$

$$
\delta_{1, j}=\left\{\begin{array}{l}
1 \\
0
\end{array}\right.
$$

and

$$
\delta_{1,1}=1, \quad \delta_{1,2}=0 .
$$

Thus, in view of (2.14a), (2.4d), (2.20), in the case described in connection with (2.23) (direction along $C_{1}$ away from $z$ ) one has

$$
\begin{aligned}
& \Phi_{0}^{+}(z)=\Phi_{0}(z)+i\left(\alpha_{1}-\alpha_{2}+2 \pi\right) F(z), \\
& \Phi_{0}^{-}(z)=\Phi_{0}(z)+i\left(\alpha_{1}-\alpha_{2}\right) F(z) ;
\end{aligned}
$$

superscripts \pm here have the same meaning as in (2.22'); similarly, when direction along $C_{1}$ is towards $z$ we have

$$
\begin{aligned}
& \Phi_{0}^{+}(z)=\Phi_{0}(z)+i\left(\alpha_{2}-\alpha_{1}\right) F(z), \\
& \Phi_{0}^{-}(z)=\Phi_{0}(z)+i\left(\alpha_{2}-\alpha_{1}-2 \pi\right) F(z) .
\end{aligned}
$$


The above is a direct consequence of (2.24).

Turning back to the general case, from (2.20) it is inferred that

$$
F(z)=\frac{1}{2 \pi i\left[\Lambda\left(\omega_{j}\right)-\Lambda\left(\omega_{s}\right)\right]}\left[\Phi_{0}^{. j}(z)-\Phi_{0}^{.0}(z)\right],
$$

$$
\Phi_{0}(z)=\frac{1}{2 \pi\left[\Lambda\left(\omega_{s}\right)-\Lambda\left(\omega_{j}\right)\right]}\left[\Phi_{0}^{. j}(z) \Omega\left(\omega_{s}\right)-\Phi_{0}^{.8}(z) \Omega\left(\omega_{j}\right)\right]
$$

whenever

$$
\Lambda\left(\omega_{s}\right) \neq \Lambda\left(\omega_{j}\right) .
$$

In the case covered by (2.21)-(2.21b) we have

$$
\begin{aligned}
& F(z)=\frac{1}{2 i \Omega\left(\omega_{j}\right)}\left[\Phi_{0}^{, j}(z)-\Phi_{0}^{, \mu+j}(z)\right], \\
& \Phi_{0}(z)=\frac{1}{2}\left[\Phi_{0}^{, j}(z)+\Phi_{0}^{, \mu+j}(z)\right]
\end{aligned}
$$

for every $j, 1 \leqq j \leqq \mu$, such that $\Omega\left(\omega_{j}\right) \neq 0$.

In Plemelj's case we have (see (M), for instance)

$$
\begin{aligned}
F(t) & =\frac{1}{2 \pi i}\left[\Phi_{0}^{+}(t)-\Phi_{0}^{-}(t)\right], \\
\Phi_{0}(t) & =\frac{1}{2}\left[\Phi_{0}^{+}(t)+\Phi_{0}^{-}(t)\right] ;
\end{aligned}
$$

these formulas are fundamental in (M), (V), (G).

Consider the case described in connection with (2.23); then (by (2.24), (2.24')). one has

$$
F(z)=\frac{1}{2 \pi i}\left[\Phi_{0}^{+}(z)-\Phi_{0}^{-}(z)\right],
$$

$$
\Phi_{0}(z)=\frac{1}{2 \pi}\left[\left(\alpha_{2}-\alpha_{1}\right) \Phi_{0}^{+}(z)+\left(2 \pi+\alpha_{1}-\alpha_{2}\right) \Phi_{0}^{-}(z)\right]
$$

when the direction along $C_{1}$ is away from $z$; if the direction along $C_{1}$ is towards $z_{\text {, }}$ (2.28) will continue to hold, but $\left(2.28^{\prime}\right)$ is to be replaced by

$$
\Phi_{0}(z)=\frac{1}{2 \pi}\left[\left(2 \pi+\alpha_{1}-\alpha_{2}\right) \Phi_{0}^{+}(z)+\left(\alpha_{2}-\alpha_{1}\right) \Phi_{0}^{-}(z)\right] .
$$

3. Continuity properties. In consequences of well known facts (cf. (M), (V) and Plemelj) we may assert that an integral (1.3), where

$$
F(y) \subset H_{k}
$$

$$
\text { (on } L ; 0<h \leqq 1 \text { ), }
$$


is of class $H_{h}$, with $H_{h}$ replaced by $H_{1-\epsilon}$ when $h=1$; this is true at all points $t$ on $L$ distinct from $z, c$-points.

Consider

$$
\Phi_{0}(x)=\int_{L_{0}} \frac{F(y) d y}{y-x},
$$

where $L_{0}$ is a suitably small portion of $L$ in the neighborhood of $z$. As in $\$ 2$, $L_{0}$ consists of curves $C_{i_{1}}, \cdots, C_{i_{\mu}}$ directed towards $z$ and curves $C_{\theta_{1}}, \cdots, C_{c_{\mu}}$ directed away. With respect to these curves we use the notation of $\$ 2$. Let $z^{\prime \prime} \neq z$ be $a$ point of $L_{0}$, say on $C_{i 1}$. By (2.4c) the principal value, in the sense of $\$ 2$,

$$
g(z)=\int_{L_{0}} \frac{d y}{y-z}
$$

is expressible as

$$
\begin{aligned}
g(z) & =\log \frac{A_{\theta_{1}} \cdots A_{\theta_{\mu}}}{A_{i_{1}} \cdots A_{i_{\mu}}}+i \Lambda, \\
\Lambda & =\left(\alpha_{i_{1}}+\cdots+\alpha_{i_{\mu}}\right)-\left(\alpha_{\theta_{1}}+\cdots+\alpha_{\theta_{\mu}}\right) .
\end{aligned}
$$

On the other hand

$$
\begin{aligned}
g\left(z^{\prime \prime}\right) & =\int_{C_{i_{1}}} \frac{d y}{y-z^{\prime \prime}}+\sum_{v=2}^{\mu} \int_{C_{i_{v}}} \frac{d y}{y-z^{\prime \prime}}+\sum_{v=1}^{\mu} \int_{C_{c_{v}}} \frac{d y}{y-z^{\prime \prime}} \\
& =\int_{C_{i_{1}}} \frac{d y}{y-z^{\prime \prime}}+\left.\sum_{v=2}^{\mu} \log \left(y-z^{\prime \prime}\right)\right|_{z+A_{t_{v}}} ^{z}+\left.\sum_{v=1}^{\mu} \log \left(y-z^{\prime \prime}\right)\right|_{z} ^{z+A_{c_{v}}}
\end{aligned}
$$

and

$$
g\left(z^{\prime \prime}\right)=\int_{C_{i_{1}}} \frac{d y}{y-z^{\prime \prime}}+\log \frac{z-z^{\prime \prime}+A_{e_{1}}}{z-z^{\prime \prime}}+\log \prod_{v=2}^{\mu} \frac{z-z^{\prime \prime}+A_{\iota_{y}}}{z-z^{\prime \prime}+A_{i_{v}}}
$$

The integral above is a principal Cauchy value in the usual sense. Thus

$$
g\left(z^{\prime \prime}\right)=q i \pi+\log \prod_{\nu=1}^{\mu} \frac{z-z^{\prime \prime}+A_{e_{\nu}}}{z-z^{\prime \prime}+A_{i_{\nu}}} \quad\left(z^{\prime \prime} \neq z\right),
$$

where $q$ is an odd integer (positive or negative) depending on the curve on which $z^{\prime \prime}$ lies. We note that $\lim g\left(z^{\prime \prime}\right)$ (as $\left.z^{\prime \prime} \rightarrow z\right)$ exists and

$$
g(z)-\lim g\left(z^{\prime \prime}\right)=i(\Lambda+q \pi) \text {. }
$$

In the sequel any z-point, for which $q$ has a value independent of the direction of approach, will be termed a $z^{\prime}$-point; z-points for which $q$ is not unique will be termed $z_{1}$-points. Examples of $z^{\prime}$-points are furnished subsequent to $\left(7.3^{\prime}\right)$.

Turning to (3.2) we obtain 
Hence

$$
\Phi_{0}(x)=\int_{L_{0}} \frac{F(y)-F(z)}{y-x} d y+F(z) g(x) .
$$

$$
\Phi_{0}\left(z^{\prime \prime}\right)-\Phi_{0}(z)=\int_{L_{0}}\left[\frac{1}{y-z^{\prime \prime}}-\frac{1}{y-z}\right][F(y)-F(z)] d y+F(z)\left[g\left(z^{\prime \prime}\right)-g(z)\right]
$$

and

$$
\begin{aligned}
& \Phi_{0}\left(z^{\prime \prime}\right)-\Phi_{0}(z)+i(\Lambda+q \pi) F(z) \\
& =\left(z^{\prime \prime}-z\right) \int_{L_{0}} \frac{F(y)-F(z)}{\left(y-z^{\prime \prime}\right)(y-z)} d y+F(z)\left[g\left(z^{\prime \prime}\right)-g(z)+i(\Lambda+q \pi)\right] .
\end{aligned}
$$

Now

$$
g\left(z^{\prime \prime}\right)-g(z)+i(\Lambda+q \pi)=\log \Pi \frac{1+\left(z-z^{\prime \prime}\right) A_{e_{p}}^{-1}}{1+\left(z-z^{\prime \prime}\right) A_{i}^{-1}} .
$$

Since $\left|z-z^{\prime \prime}\right|\left|A_{j}^{-1}\right|<1$ one obtains

$$
\left|g\left(z^{\prime \prime}\right)-g(z)+i(\Lambda+q \pi)\right|<c^{*}\left|z^{\prime \prime}-z\right| \text {. }
$$

Let $y_{j}$ be a point on $C_{j}$ such that

$$
\left|y_{j}-z\right|=2\left|z-z^{\prime \prime}\right| \quad(j=1, \cdots, 2 \mu) ;
$$

we take $2\left|z-z^{\prime \prime}\right|<A$. We write

$$
L_{0}=L_{1}+L_{2},
$$

where $L_{1}$ is the part of $L_{0}$ at distance not greater than $2\left|z-z^{\prime \prime}\right|$ from $z_{0}$

For $y$ in $L_{2}$ one has

$$
|y-z| \geqq 2\left|z-z^{\prime \prime}\right|
$$

consequently

$$
\left|\frac{y-z}{y-z^{\prime \prime}}\right| \leqq 1+\left|\frac{z^{\prime \prime}-z}{y-z^{\prime \prime}}\right| \leqq 2
$$

Accordingly, writing

we obtain

$$
\begin{aligned}
\Gamma_{2} & \equiv\left(z^{\prime \prime}-z\right) \int_{L_{2}} \frac{F(y)-F(z)}{\left(y-z^{\prime \prime}\right)(y-z)} d y \\
& =\left(z^{\prime \prime}-z\right) \int_{L_{2}} \frac{y-z}{y-z^{\prime \prime}} \frac{F(y)-F(z)}{(y-z)^{2}} d y,
\end{aligned}
$$

$$
\left|\Gamma_{2}\right| \leqq c^{*}\left|z^{\prime \prime}-z\right| \int_{L_{2}}|y-z|^{h-2}|d y|
$$


Now $|d y| \leqq c^{*} d|y-z| ;$ hence

$$
\left|\Gamma_{2}\right| \leqq c^{*}\left|z^{\prime \prime}-z\right| \int_{2\left|z^{\prime}-z\right|}^{A} u^{h-2} d u=c^{*}\left|z^{\prime \prime}-z\right|\left[-A^{h-1}+2^{h-1}\left|z^{\prime \prime}-z\right|^{h-1}\right]
$$

and

$$
\left|\Gamma_{2}\right|<c^{*}\left|z^{\prime \prime}-z\right|^{h}
$$

provided $h<1$; when $h=1$ one has

(3.5a) $\left|\Gamma_{2}\right| \leqq c^{*}\left|z^{\prime \prime}-z\right| \log \frac{A}{2\left|z-z^{\prime \prime}\right|} \leqq c^{*}\left|z^{\prime \prime}-z\right| \log \frac{1}{\left|z^{\prime \prime}-z\right|} \cdot$

Considering the integral along $L_{1}$ we put

$$
\begin{aligned}
\Gamma_{1}= & \int_{L_{1}}\left[\frac{1}{y-z^{\prime \prime}}-\frac{1}{y-z}\right][F(y)-F(z)] d y=\int_{L_{1}} \frac{F(y)-F\left(z^{\prime \prime}\right)}{y-z^{\prime \prime}} d y \\
& -\int_{L_{1}} \frac{F(y)-F(z)}{y-z} d y+\left[F\left(z^{\prime \prime}\right)-F(z)\right] \int_{L_{1}} \frac{d y}{y-z^{\prime \prime}} .
\end{aligned}
$$

The integral last displayed is expressible by the formulas subsequent to (3.3), provided $C_{j}$ is replaced by the part of $C_{j}$ from $z$ to $y_{i}(j=1, \cdots, 2 \mu)$; thus, replacing $z+A_{i}$ in (3.3a) by $y_{j}$ we obtain

$$
\int_{L_{1}} \frac{d y}{y-z^{\prime \prime}}=-q i \pi+\log \prod_{\prod_{1}}^{\mu} \frac{y_{e_{0}}-z^{\prime \prime}}{y_{i_{p}}-z^{\prime \prime}} .
$$

Since the $y_{j}$ are points on a circle with center at $z$ and radius $2\left|z^{\prime \prime}-z\right|$ it follows that

$$
\left|z^{\prime \prime}-z\right| \leqq\left|y_{j}-z^{\prime \prime}\right| \leqq 3\left|z^{\prime \prime}-z\right|
$$

hence

$$
\left|\frac{y_{\theta_{v}}-z^{\prime \prime}}{y_{i_{v}}-z^{\prime \prime}}\right| \leqq 3
$$

accordingly it is easily concluded that

$$
\left|\int_{L_{1}} \frac{d y}{y-z^{\prime \prime}}\right| \leqq c^{*} .
$$

For the second integral in the third member of (3.6), we obtain immediately that

$$
\left|\int_{L_{1}} \frac{F(y)-F(z)}{y-z} d y\right| \leqq c^{*} \int_{0}^{2\left|z^{\prime \prime}-z\right|}|y-z|^{n-1} d|y-z|<c^{*}\left|z^{\prime \prime}-z\right|^{n}
$$

since $|d y| \leqq c^{*} d|y-z|$ along each $C_{v}$. A similar inequality may be established 
for the first integral, since $|d y|\left\langle c^{*} d\left|y-z^{\prime \prime}\right|\right.$ along $C_{i}$, and since $3\left|z^{\prime \prime}-z\right|$ $\geqq\left|y-z^{\prime \prime}\right| \geqq \gamma\left|z^{\prime \prime}-z\right|(\gamma>0)$ and

$$
\left|F(y)-F\left(z^{\prime \prime}\right)\right|<c^{*}\left(|y-z|^{h}+\left|z^{\prime \prime}-z\right|^{h}\right)<c^{*}\left|z^{\prime \prime}-z\right|^{h}
$$

for $y$ on $L_{1} C_{i}\left(j \neq i_{1}\right)$. These inequalities hold for $0<h \leqq 1$. In view of the results just obtained and of (3.6a), (3.6) it is inferred that

$$
\left|\Gamma_{1}\right|<c^{*}\left|z^{\prime \prime}-z\right|^{h} \quad(0<h \leqq 1) ;
$$

whence by (3.5), (3.5a)

$\left|\left(z^{\prime \prime}-z\right) \int_{L_{0}} \frac{F(y)-F(z)}{\left(y-z^{\prime \prime}\right)(y-z)} d y\right|=\left|\Gamma_{1}+\Gamma_{2}\right|<c^{*}\left|z^{\prime \prime}-z\right|^{h}(0<h<1)$

and

$$
\left|\Gamma_{1}+\Gamma_{2}\right|<c^{*}\left|z^{\prime \prime}-z\right| \log \frac{1}{\left|z^{\prime \prime}-z\right|}
$$

when $h=1$. Finally (by (3.4) and (3.4a)), we get the formulas

$$
\left|\Phi_{0}\left(z^{\prime \prime}\right)-\Phi_{0}(z)+i(\Lambda+q \pi) F(z)\right|<c^{*}\left|z^{\prime \prime}-z\right|^{n}
$$

if $h<1$, and

$$
\left|\Phi_{0}\left(z^{\prime \prime}\right)-\Phi_{0}(z)+i(\Lambda+q \pi) F(z)\right|<c^{*}\left|z-z^{\prime \prime}\right| \log \frac{1}{\left|z-z^{\prime \prime}\right|}
$$

if $h=1$; here $q$, as indicated above, is an odd integer depending on the curve on which $z^{\prime \prime}$ lies; $\Phi_{0}(z)$ is principal value (in the extended sense) of

$$
\Phi_{0}(x)=\int_{L_{0}} \frac{F(y) d y}{y-x}
$$

at a z-point, while $\Phi_{0}\left(z^{\prime \prime}\right)$ is the principal value (in the usual sense) of $\Phi_{0}(x)$ at any point $z^{\prime \prime}$, not equal to $z$, of $L_{0}$. These formulas will obviously hold also when $L_{0}$ is replaced by $L$.

A corollary of (3.7) $-(3.7 \mathrm{~b})$ is that the statement in the opening paragraph of this section can be modified by the assertion that, if $h=1$, the principal value $\Phi_{0}(t)$ of $\Phi_{0}(x)$ satisfies the inequality

$$
\left|\Phi_{0}(t)-\Phi_{0}\left(t^{\prime}\right)\right|<c^{*}\left|t-t^{\prime}\right| \log \frac{1}{\left|t-t^{\prime}\right|}
$$

for $t^{\prime}$ (on $L_{0}$ ) in the neighborhood of $t$, not equal to $z$, on $L_{0}$.

We also note that a consequence of (3.7), (3.7a) is as follows. The function $\Phi_{0}^{*}(t)$, such that

$$
\Phi_{0}^{*}(t)=\Phi_{0}(t) \quad\left(t \text { on } L_{0} ; t \neq z\right),
$$




$$
\Phi_{0}^{*}(z)=\lim \Phi_{0}(t)
$$

(as $t \rightarrow z$ along $L_{0}$ ),

is continuous on $L_{0}$, except at $z_{1}$-points; at $z_{1}$-points the discontinuities are of the first kind and the limits along the various curves are attained with a degree of continuity indicated in (3.7), (3.7a); also, the various limits at a point $z_{1}$ are furnished by (3.7), (3.7a). If $h<1, \Phi_{0}^{*}(t) \subset H_{h}$ and if $h=1, \Phi_{0}^{*}(t) \subset H^{(\log )}$ on $L_{0}$, $z_{1}, c$-points excepted. In this connection the designation

$$
f(t) \subset H^{(\log )}
$$

signifies that

$$
\left|f\left(t^{\prime}\right)-f\left(t^{\prime \prime}\right)\right|<c^{*}\left|t^{\prime}-t^{\prime \prime}\right| \log \frac{1}{\left|t^{\prime}-t^{\prime \prime}\right|} .
$$

In the sequel $a$ "star," used for a superscript, will often designate that there is at hand a function which at points of $L$ (or $L_{0}$ or any set involved) distinct from z-points and c-points is equal to a Cauchy principal value (in the ordinary sense) and which at every $z^{\prime}$-point is defined as the limit of the principal value.

In view of the statements with respect to (3.8), (3.8a) every "starred" function originating from a Cauchy principal value is continuous on $L$ (or $\left.L_{0}\right)$, $z_{1}$ excepted; at all points of $L$ (or $\left.L_{0}\right), z_{1}, c$-points possibly excepted, such a function is $H_{h}$, if the numerator in the integrand is $H_{h}(h<1)$, and $H^{(\log )}$ if the nu-. merator in the integrand is $H_{1}$.

In the sequel functions will be also "starred" in the extended sense as follows. A designation $\phi^{*}(t)$, for instance, would mean that $\phi^{*}(t)=\phi(t)$ on $L$, except at $z$-points, and that $\phi^{*}(z)=\lim \phi(t)$ (as $t \rightarrow z$ along $L$ at $z$ points at which the discontinuities of $\phi$ are removable); the function $\phi(t)$ does not need to be a Cauchy principal value.

In consequence of (3.7), (3.7a) the following may be asserted. If $\phi^{*}(t)$ can be formed and is of a class $H_{h}$ then, on writing

$$
\Phi(t)=\int_{L} \frac{\phi^{*}(y) d y}{y-t}
$$

we have

$$
\Phi^{*}(z)=\Phi(z)-i(\Lambda+q \pi) \phi^{*}(z)
$$

at every $z$-point; $\Lambda+q \pi$ is unique at every $z^{\prime}$-point.

In order to handle with sufficient precision the situation near $c$-points we adapt the definition of classes $H^{*}$, introduced in $(\mathrm{M})$; a function $\phi(t)$ is of class $H^{*}$ if $\phi(t)$ is of class $H_{h}$ in every closed subset of $L$ not containing points $z_{1}, c$ (but possibly containing $z^{\prime}$-points), while in a neighborhood of $c$ one has

$$
\phi(t)=\frac{f_{1}(t)}{(t-c)^{\alpha}} \quad(0<\alpha<1),
$$


where $f_{1}(t) \subset H_{p}$ near and at $t=c,|\phi(t)||d t|$ being integrable over $L$.

We conclude this section with the following from $(M ; 5)$. If $\phi(t)$ is of a class $H_{h}$ ( $c$ included) and $\phi(c)=0$ then for $\Phi(t)$ of (3.9) one has

$$
\Phi^{+}(c)=\Phi^{-}(c)=\Phi(c)=\int_{L} \frac{\phi(t) d t}{t-c}
$$

if $\phi(c) \neq 0$, then

$$
\Phi(x)=\mp \phi(c) \log (x-c)+\Phi_{1}(x)
$$

(a suitable branch of $\log \cdots$ ), where $\Phi_{1}(x)$ is bounded near $c$ and $\lim \Phi_{1}(x)$ exists and is unique as $x \rightarrow c$ along any path; (3.11a) holds also for $t$ on $L$ near $c$. When $\phi(t) \subset H^{*}$ (cf. (3.10)) then near $c$ one has

$$
\Phi(x)= \pm \frac{f_{1}(c) \pi \exp ( \pm i \alpha \pi)}{\sin \alpha \pi(x-c)^{\alpha}}+O\left(|x-c|^{-(\alpha-\beta)}\right)
$$

(some positive $\beta$ ). In (3.11a), (3.11b) the upper (lower) sign is used when the direction along $L$ is away from (towards) $c$. In particular

$$
\Phi(t)= \pm \pi \cot \alpha \pi \frac{f_{1}(c)}{(t-c)^{\alpha}}+O\left(|t-c|^{-(\alpha-\beta)}\right)
$$

for $t$ on $L(t \neq z)$.

In the sequel, unless the contrary is stated, the equations involved will be solved except at the c-points and z-points.

It will be said that a function $f(t)$ is logarithmically infinite at $z$ if $f(t)$ $=O(\log (1 /|t-z|))$ near $z$.

4. Preliminary boundary problems. With a direction assigned along every part of $L$, every arc or curve constituting $L$ will have a + and a - side; in this connection, the $+(-)$ side will be the side on the left (right) when facing along the curve.

We observe that

$$
L=L^{0}+\Omega,
$$

where $L^{0}$ consists of closed curves only, while $\Omega$ consists of open arcs and contains no closed curves. $\Omega$ may have points in common with $L^{0}$; the complement of $\Omega$ is connected.

$L^{0}$ consists of $\mu$ disjoint portions $L_{j}(j=1, \cdots, \mu)$, where $L_{j}$ is connected (consists of closed curves). Let $C\left(L_{j}\right)$ be the open set complementary to $L_{j}$; one has

$$
C\left(L_{j}\right)={ }_{j} \mathrm{O}_{1}+{ }_{j} \mathrm{O}_{2}+\cdots+{ }_{j} \mathrm{O}_{c j} \text {, }
$$

where ${ }_{j} O$, is open connected; for a fixed $j$ no two sets ${ }_{j} O,(\nu=1,2, \cdots)$ have points in common; ${ }_{j} O_{c_{j}}$ is to be the set containing the point infinity. Of course

$$
C\left(L_{1}\right) \cdots C\left(L_{\mu}\right)=C\left(L^{0}\right), \quad L^{0}=L_{1}+\cdots+L_{\mu} .
$$


DEFINITION 4.1. $\Phi(x)$ will be said to be regular in $C\left(L_{j}\right)$ if

$$
\Phi(x)={ }_{j} \Phi_{v}(x)
$$

$\nu=1, \cdots, c_{j}$, where ${ }_{j} \Phi_{\nu}(x)$ is analytic in ${ }_{j} O_{\nu}$ except, perhaps, for a pole, at a point ${ }_{j} d_{v}$ of ${ }_{j} O_{r ;},{ }_{j} d_{c_{j}}=\infty$.

Now $C\left(L^{0}\right)$ has a decomposition

$$
C\left(L^{0}\right)=O_{1}+O_{2}+\cdots+O_{\mu},
$$

into open connected sets $O_{\nu} ; O_{\mu^{\prime}}$ contains $\infty$. If ${ }_{j} \Phi(x)$ is regular in $C\left(L_{j}\right)$ the function

$$
\Phi(x)={ }_{1} \Phi(x) \cdots{ }_{\mu} \Phi(x)
$$

will be analytic in $C\left(L^{0}\right)$ except, perhaps, for a finite number of poles. If the location of poles ${ }_{j} d_{\nu}$ of ${ }_{j} \Phi(x)$ is at our disposal then suitably choosing the ${ }_{j} d_{\nu}$ we can always arrange so that $\Phi(x)$ has at most one pole in $O,\left(\nu=1, \cdots, \mu^{\prime}\right)$. Thus, for a suitable choice of the ; $d_{\text {, }}$ the function $\Phi(x)(4.4)$ is regular in $C\left(L^{0}\right)$.

We consider now the problem

$$
\psi_{0}^{+}(t)=A(t) \overline{\psi_{0}}(t)
$$

where $A(t) \subset H_{p}$ on $L$ including $z, c$-points and

$$
A(t) \neq 0
$$

(on $L, c$-points included).

We seek to satisfy (4.5) by a function $\psi_{0}(x)$ regular in $C\left(L^{0}\right)$ and having as few poles in $C\left(L^{0}\right)$ as possible; moreover, the solution is to be such that

$$
\psi_{0}^{+}(t) \neq 0
$$

(on $L, c$-points included).

Suppose ${ }_{j} \psi(x)$ is a solution of

$$
{ }^{*} \psi^{+}(t)=A(t), \psi^{-}(t)
$$

regular in $C\left(L_{i}\right)$ and such that

$$
s^{+}+(t) \neq 0
$$

On $L_{\nu}(v \neq j)$ of course $j \psi^{+}(t)={ }_{j} \psi^{-}(t)={ }_{j} \psi(t)$. Provided the poles ${ }_{j} d_{\nu}(\nu=1$, $\cdots, c_{j}$ ) of $\psi(x)$ can be suitably chosen (not on $L$ ), the function

$$
\psi_{0}(x)={ }_{1} \psi(x) \cdots{ }_{\mu} \psi(x)
$$

will be regular in $C\left(L^{0}\right)$; moreover, $\psi_{0}(x)$ will satisfy (4.5), while

$$
\psi_{0}^{+}(t)={ }_{1} \psi^{+}(t) \cdots{ }_{\mu} \psi^{+}(t) \neq 0
$$

in consequence of $\left(4.6^{\prime}\right)$. We shall now carry out the indicated plan of solving (4.5).

$L_{i}$ may involve a number of loops, $l_{j, 1}, l_{i, 2}, \ldots$. As can be seen from earlier 
literature, relating to more special cases, of importance in connection with any loop $l$ is the "index" of $A$. We shall write

$$
\text { ind. }(l, A)=\frac{1}{2 \pi i} \int_{l} d \log A \text {. }
$$

Here integration is in the counterclockwise direction. We form "indices" only for loops. Clearly ind. $(l, A)$ is an integer $(0$, greater than 0 , or less than 0$)$. We determine indices

$$
\text { ind. }\left(l_{j, \nu}, A\right)=n_{j, \nu} \quad(\nu=1,2, \cdots) .
$$

On taking account of the $n_{i, v}$ and of the topology of $L_{i}$ we form a "uniformizing" function

$$
u_{j}(x)=\left(x-{ }_{j} d_{1}\right)^{m_{j, 1}}\left(x-{ }_{j} d_{2}\right)^{m_{j, 2}} \cdots\left(x-j_{j} d_{c^{\prime}}\right)^{m_{j, 0^{\prime}}}
$$

$\left(c^{\prime}=c_{j}-1\right)$, where the $m_{j,}$ are suitable integers and ${ }_{j} d_{v}$ is any point in ${ }_{j} O_{v}$ (cf. (4.1)); the function is to have the property that

$$
\log \left[A(t) u_{j}^{-1}(t)\right]
$$

is uniform on $L_{j}$. As to the $m_{j, v}$, we note that

$$
m_{j, \nu}=\text { ind. }\left(F\left(, O_{v}\right), A\right) \text {, }
$$

where

$$
F\left(, O_{v}\right)
$$

is the frontier of ${ }_{j} \mathrm{O}_{r}$. We observe that

$$
s^{+} \psi^{+}(t)=u_{j}(t) A_{j}(t), \psi^{-}(t)
$$

where

$$
A_{j}(t)=A(t) u_{j}^{-1}(t)
$$

is of a Hölder class on $L, c$-points included, $A_{j}(t) \neq 0$ on $L, c$-points included. We shall seek a solution of (4.10) in the form

$$
{ }_{j} \psi(x)=G_{j}(x) \Gamma_{j}(x),
$$

where $G_{j}(x), \Gamma_{j}(x)$ satisfy

$$
\begin{aligned}
G_{j}^{+}(t) & =u_{j}(t) \overline{G_{j}}(t) \\
\Gamma_{j}^{+}(t) & =A_{j}(t) \overline{\Gamma_{j}}(t)
\end{aligned}
$$

respectively.

Since $\log A_{j}(t)$ is uniform on $L_{i}$, a solution of (4.11b) can be given in the form

$$
\Gamma_{j}(x)=\exp \left\{\frac{1}{2 \pi i} \int_{L_{j}} \frac{\log A_{j}(y) d y}{y-x}\right\}
$$


this is asserted for all points of $L_{j}$, except for $z$-points, and is established with the aid of the original Plemelj formulas (cf. $\left(2.22^{\prime}\right)$ ); $\Gamma_{j}(x)$ is analytic in $C\left(L_{j}\right)$ and does not vanish in $C\left(L_{j}\right) ; \Gamma_{j}(\infty)=1$. Inasmuch as $L_{v}$ is in $C\left(L_{j}\right)$ for $v \neq j$, we have

$$
\Gamma_{j}^{+}(t)=\Gamma_{j}(t) \neq 0
$$

(on $L_{v}, \nu \neq j, z$-points of $L_{v}$ included).

On the other hand,

$$
\Gamma_{j}^{+}(t)=\exp \left\{\frac{1}{2 \pi i} \int_{L_{j}} \frac{\log A_{j}(y) d y}{y-t}\right\}_{j}^{1 / 2}(t)
$$

$A_{i}^{1 / 2}(t) \neq 0, \neq \infty$ on $L_{j}$ (z-points included); the exponential factor is finite and does not vanish on $L_{j}$, except perhaps at $z$-points. In view of (3.9), (3.9a) at a $z^{\prime}$-point of $L_{j}$ we have

$$
\begin{gathered}
\Gamma_{j}^{+*}\left(z^{\prime}\right)=A_{j}^{1 / 2}\left(z^{\prime}\right) \exp \{\cdots\}^{*}=A_{j}^{1 / 2}\left(z^{\prime}\right)\left[\operatorname { e x p } \left(\frac{1}{2 \pi i} \int_{L_{j}} \frac{\log A_{j}(y) d y}{y-z^{\prime}}\right.\right. \\
\left.-\left(\frac{\Lambda+q \pi}{2 \pi}\right) \log A_{j}\left(z^{\prime}\right)\right] \\
=A_{j}^{1 / 2-(1 / 2 \pi)(\Delta+q \pi)}\left(z^{\prime}\right) \exp \left(\frac{1}{2 \pi i} \int_{L_{j}} \frac{\log A_{j}(y) d y}{y-z^{\prime}}\right) .
\end{gathered}
$$

Here the integral last displayed is a "Cauchy principal value" in the extended sense; $\Lambda+q \pi$ depends on $z^{\prime}$. Accordingly

$$
\Gamma_{j}^{+*}(t) \neq 0, \neq \infty,
$$

for $t$ on $L_{j}$. In this connection we see the importance of uniformity of $\log A_{j}(y)$ on $L_{j}$, since in the contrary case existence of unique limit $\Gamma_{i}^{+*}\left(z^{\prime}\right)$ could not be asserted.

To solve (4.11a) we first form a function

$$
v_{j}(x)=\left(x-{ }_{j} d_{1}\right)^{s_{j, 1}}\left(x-{ }_{j} d_{2}\right)^{s_{j, 2}} \cdots\left(x-{ }_{j} d_{c^{\prime}}\right)^{s_{j, c^{\prime}}} \quad\left(c^{\prime}=c_{j}-1\right)
$$

(cf. (4.9a)) where the $s_{i, 1}$ are arbitrary integers. We recall the decomposition (4.1) of $C\left(L_{j}\right) ;{ }_{j} O_{c_{j}}$ is an open set containing infinity. We put

$$
G_{j}(x)=v_{j}(x)
$$

We obtain in a unique manner

$$
G_{j}(x)=v_{j}(x) u_{j}(x)^{t j, \nu}
$$$$
\text { (in }{ }_{j} O_{\nu} ; \nu=1, \cdots, c_{j} \text { ), }
$$

where the $t^{i, v}$ are certain integers; $t^{j, c_{j}}=0$. We observe that

$$
G_{j}(x), \quad G_{j}^{-1}(x)
$$

are regular in $C\left(L_{j}\right)$ with possible poles at the ${ }_{j} d_{\nu}(\nu=1,2, \cdots)$, these points 
representing the only possible poles in $C\left(L_{j}\right)$.

We shall give a few typical examples of determination of $G_{j}(x)$ which could be used as a model to follow in applications.

Suppose $L_{j}$ consists of simple closed curves $S_{1}, S_{2}, S_{3}$, intersecting in just four points (z-points), $z_{1}, z_{2}, z_{3}, z_{4}\left(z_{3}\right.$ interior $\left.S_{2}\right), z_{1}$ being the intersection of $S_{1}, S_{2}, S_{3}, z_{2}$ of $S_{1}, S_{2}, z_{3}$ of $S_{1}, S_{3}, z_{4}$ of $S_{2}, S_{3}$. With $S_{1}{ }^{0}, S_{2}{ }^{0}, S_{3}{ }^{0}$ denoting the domains interior to $S_{1}, S_{2}, S_{3}$, respectively, we define $O_{1}, \cdots, O_{7}$ as follows :

$$
\begin{aligned}
& O_{1}=\left\{S_{1}^{0}-S_{2}^{0}\right\}, \quad O_{2}=\left\{S_{1}^{0} S_{2}^{0}-S_{3}^{0}\right\}, \quad O_{3}=\left\{S_{2}^{0}-S_{1}^{0}-S_{3}^{0}\right\}, \\
& O_{4}=\left\{S_{1}^{0} S_{3}^{0}\right\}, \quad O_{6}=\left\{S_{2}^{0} S_{3}^{0}-S_{1}^{0}\right\}, \quad O_{6}=\left\{S_{3}^{0}-S_{2}^{0}\right\}, \\
& O_{7}=\left\{C\left(S_{1}^{0}+S_{2}^{0}+S_{3}^{0}\right)\right\} \text {; }
\end{aligned}
$$

here braces indicate that frontier points of the sets involved are discarded. $O_{1}, \cdots, O_{7}$ are bounded by arcs of

$\left(S_{1}, S_{2}\right), \quad\left(S_{1}, S_{2}, S_{3}\right), \quad\left(S_{1}, S_{2}, S_{3}\right), \quad\left(S_{1}, S_{3}\right), \quad\left(S_{1}, S_{2}, S_{3}\right), \quad\left(S_{2}, S_{3}\right), \quad\left(S_{1}, S_{2}, S_{3}\right)$,

respectively. Suppose, for instance, that directions along $S_{1}, S_{2}$ are counterclockwise and along $S_{3}$ clockwise. Along each arc involved, we accordingly fix the positive and negative side. In $O_{7}$ in accordance with (4.14) we put $G_{j}(x)=v_{j}(x)$. From $O_{7}$ we proceed "inward," noting that in order to satisfy (4.11a), the value on the negative side is multiplied by $u_{j}$ and that on the positive side by $u_{j}^{-1}$. Accordingly

$$
\left.\left.G_{j}=u_{j} v_{j} \quad \text { (in } O_{1}\right), \quad G_{j}=u_{j} v_{j} \quad \text { (in } O_{3}\right), \quad G_{j}=u_{j}^{-1} v_{j} \text { (in } O_{6} \text { ). }
$$

Using these relations we perform the next step, proceeding further "inward," obtaining

$$
G_{j}=u_{j}^{2} v_{j} \quad\left(\text { in } O_{2}\right), \quad G_{j}=v_{j} \quad\left(\text { in } O_{5}\right) .
$$

Whence one more step will yield

$$
G_{j}=u_{j} v_{j}
$$

Thus $G_{j}(\dot{x})$ has been determined in the form (4.15) in $C\left(L_{j}\right)$.

Suppose, now, that $L_{j}$ consists of simple closed curves $S_{1}, S_{2}, S_{3}\left(S_{2}\right.$ inside $S_{1}$ and $S_{3}$ inside $S_{2}$ ), having a single point, $z$, in common; let the direction be counterclockwise along $S_{1}, S_{2}$ - clockwise along $S_{3}$. Let $S_{1}{ }^{0}, S_{2}{ }^{0}, S_{3}{ }^{0}$ be regions interior to $S_{1}, S_{2}, S_{3}$, respectively. We define domains

$$
O_{1}=S_{3}^{0}
$$$$
\mathrm{O}_{2}=\left\{S_{2}^{0}-S_{3}^{0}\right\}
$$$$
O_{3}=\left\{S_{1}^{0}-S_{2}^{0}\right\},
$$$$
O_{4}=\left\{C\left(S_{1}^{0}\right)\right\}
$$

The solution, which in $O_{4}$ has the value $v_{j}(x)$, will be

$$
\left.\left.G_{j}=u_{j} v_{j} \quad \text { (in } O_{3}\right), \quad G_{j}=u_{j}^{2} v_{j} \quad \text { (in } O_{2}\right), \quad G_{j}=u_{j} v_{j} \text { (in } O_{1} \text { ). }
$$


In general, it can be seen that the integers $t^{i, v}$ may be determined as follows. Let

$$
T_{j}(x)=\frac{1}{2 \pi i} \int_{L_{j}} \frac{d y}{y-x} .
$$

It is seen that $T_{j}(x)$ is constant and equal to an integer over each of the regions ${ }_{j} O_{v}$, being zero in ${ }_{j} O_{c_{j}}$. Moreover (from $\left(2.22^{\prime}\right)$ )

$$
T_{j}^{+}(t)-T_{j}^{-}(t)=1
$$

Thus it follows that $t^{i, v}=T_{j}(x)$ for $x$ on ${ }_{j} O_{\nu}$.

In view of (4.7), (4.11), (4.15), (4.12) the problem (4.5) has a solution

$$
\psi_{0}(x)=G_{1}(x) \cdots G_{\mu}(x) \exp \left\{\frac{1}{2 \pi i} \sum_{j=1}^{\mu} \int_{L_{j}} \frac{\log A_{j}(y) d y}{y-x}\right\} .
$$

The ${ }_{j} d_{\nu}(j, \nu=1,2, \cdots)$ will be assumed to be chosen so that

$$
G_{1}(x) \cdots G_{\mu}(x)
$$

has in $O_{s}$ (cf. decomposition (4.3)) at most one pole $d_{s .}$ Accordingly the functions

$$
\psi_{0}(x), \quad \psi_{0}^{-1}(x)
$$

are regular in $C\left(L^{0}\right)$. One has

$$
\psi_{0}^{+}(t) \neq 0, \quad \neq \infty
$$

moreover, $\psi_{0}^{+*}\left(z^{\prime}\right)$ exists as a unique limit,

$$
\psi_{0}^{+*}\left(z^{\prime}\right) \neq 0, \quad \neq \infty,
$$

at those $z^{\prime}$-points of $L^{0}$ at which $G_{1}{ }^{+}(t) \cdots G_{\mu}+(t)$ is continuous.

With $\Omega$ denoting the set introduced at the beginning of this section consider the problem

$$
\theta^{+}(t)=A(t) \theta^{-}(t)
$$

where $A(t)$ is as in (4.5). This we solve by the method given in (M). Thus we put

$$
\theta(x)=\gamma(x) \exp \left\{\frac{1}{2 \pi i} \int_{\mathrm{a}} \frac{\log A(y) d y}{y-x}\right\}
$$

for $x$ in $C(\Omega)$; here, on taking account of the text at the end of $\S 3$, we let

$$
\gamma(x)=\prod_{c}(x-c)^{\kappa(c)},
$$

where the product is over all the $c$-points of $L$ and $k(c)$, for a fixed $c$-point, is the greatest integer such that 


$$
\kappa(c) \leqq-\alpha(c), \quad \alpha(c)=R\left(\mp \frac{\log A(c)}{2 \pi i}\right) .
$$

In $\left(4.17 b^{\prime}\right)$ the sign is to be chosen as specified subsequent to (3.11b). One has $\left(4.17 \mathrm{~b}^{\prime \prime}\right)$

$$
-1<\alpha(c)+\kappa(c) \leqq 0
$$

and

$$
\begin{aligned}
\left|\theta^{+}(t)\right| & =\left|A^{1 / 2}(t)\right| \prod_{c}|t-c| \kappa(c)\left|\exp \left\{\frac{1}{2 \pi i} \int_{\Omega} \frac{\log A(y) d y}{y-t}\right\}\right| \\
& =e^{\beta^{\prime}(t)}|t-c|^{\alpha(c)+\kappa(c)}
\end{aligned}
$$

for $t$ on $\Omega$ in a vicinity of a $c$-point $\left(\beta^{\prime}(t)\right.$ bounded). Whence

$$
\left|\theta^{+}(t)\right|^{-1}=e^{-\beta^{\prime}(t)}|t-c|^{p(c)}
$$$$
(0 \leqq \nu(c)<1)
$$

on $\Omega$ near $c$-points of $\Omega$. Using (3.9), (3.9a) and the fact that $\log A$ is uniform on $\Omega$ we prove existence of unique limits (as $t \rightarrow a z^{\prime}$-point of $\Omega$ along $\Omega$ ) $\theta^{+*}\left(z^{\prime}\right)$; one has

$\left(4.17 c^{\prime \prime}\right)$

$$
\theta^{+*}\left(z^{\prime}\right) \neq 0, \quad \neq \infty .
$$

Away from $z^{\prime}$ - and $c$-points of $\Omega$ we have on $\Omega$

$\left(4.17 c^{\prime \prime \prime}\right)$

$$
\theta^{+}(t) \neq 0, \quad \neq \infty
$$

$\theta(x)$ is "regular" in $C(\Omega)$; moreover,

$$
\theta(x) \neq 0, \quad \neq \infty
$$

in $C(\Omega)$ except, perhaps, at $x=\infty$.

Consideration of the solutions, found above, of the equations (4.5), (4.17) leads to the conclusion that the problem

$$
\psi^{+}(t)=A(t) \psi^{-}(t)
$$

has a solution, except perhaps at $z, c$-points, of the form

$$
\psi(x)=\theta(x) \psi_{0}(x)
$$

for $x$ in $C(L)$; here $\theta(x)$ is from (4.17a), (4.17b), $\left(4.17 \mathrm{~b}^{\prime}\right)$ and $\psi_{0}(x)$ is defined in (4.16), (4.15), (4.9a, c), (4.13), (4.10').

The solution is "regular" in $C(L)$; choosing the $s_{j, v}$ in (4.13) suitably one can arrange to have $x=\infty$ as a zero or as a pole of any preassigned order.

We consider now the nonhomogeneous problem

$$
\Phi^{+}(t)=A(t) \Phi^{-}(t)+B(t)
$$

where $A(t)$ is $H_{p}$ on $L, A(t) \neq 0$ on $L\left(z, c\right.$-points included) and where $B(t)$ is $H_{p}$ on $L$ ( $z, c$-points included). We put

$$
\Phi(x)=\psi(x) \Phi_{0}(x),
$$


with $\psi(x)$ given by (4.18a). Substitution in (4.19) will yield

$$
\Phi_{0}^{+}(t)-\Phi_{0}^{-}(t)=B(t) \psi^{+}(t)^{-1} \equiv C_{0}(t)
$$

$C_{0}(t)$ is of a Hölder class on $L$ except perhaps at $z, c$-points. By (4.16b), $\left(4.17 \mathrm{c}^{\prime \prime \prime}\right)$

$$
C_{0}(t) \neq 0, \quad \neq \infty
$$

(on $L_{0}$, except at $z$-points).

In view of $(4.16 \mathrm{c}),\left(4.17 \mathrm{c}^{\prime \prime}\right)$ the limits

$$
C^{*}\left(z^{\prime}\right)=B\left(z^{\prime}\right) \psi_{0}^{+*}\left(z^{\prime}\right)^{-1} \theta^{+*}\left(z^{\prime}\right)^{-1} \neq \infty
$$

exist at $z^{\prime}$-points of $L^{0}$, which are not on $\Omega$, at which $G_{1}{ }^{+}(t) \cdots G_{\mu}{ }^{+}(t)$ is continuous, and at $z^{\prime}$-points of $\Omega$ which are not on $L^{0}$; at the former points $\theta(x)$ is analytic so that

$$
\theta^{+*}\left(z^{\prime}\right)=\theta^{+}\left(z^{\prime}\right)=\theta\left(z^{\prime}\right) \neq 0, \quad \neq \infty
$$

and we use (4.16c); at the latter points $\psi_{0}(x)$ is analytic, one has

$$
\psi_{0}^{+*}\left(z^{\prime}\right)=\psi_{0}^{+}\left(z^{\prime}\right)=\psi_{0}\left(z^{\prime}\right) \neq 0, \quad \neq \infty
$$

and use is made of $\left(4.17 \mathrm{c}^{\prime \prime}\right)$. At z-points of $L$, which are the points common to $L^{0}$ and $\Omega$, or at which $G_{1}+\ldots G_{\mu}+$ is not continuous, generally $\left(4.20 \mathrm{a}^{\prime}\right)$ cannot be asserted; in fact, $C^{*}(z)$ cannot be formed.

A solution of $(4.20 \mathrm{a})$ on $L$, except perhaps at $z, c$-points of $L$, analytic in $C(L)$ and such that

$$
\Phi_{0}(x)=O\left(x^{N}\right) \quad(\text { integer } N \geqq 0 ; \text { at } \infty),
$$

may be given by

$$
\Phi_{0}(x)=\frac{1}{2 \pi i} \int_{L} \frac{B(y) \psi^{+}(y)^{-1} d y}{y-x}+P_{N}(x)
$$

for $x$ in $C(L)$, where $P_{N}(x)$ is a polynomial of degree not greater than $N$. In view of the preceding developments $B(y) \psi^{+}(y)^{-1}$ is bounded on $L$, is of a Hölder class on $L$ except, perhaps, at $z, c$-points of $L$, has removable discontinuities at $z^{\prime}$-points, as described subsequent to $\left(4.20 \mathrm{a}^{\prime}\right)$, and, generally, has irremovable discontinuities of the first kind at the other z-points; obviously $B(y) \psi^{+}(y)^{-1}$ is continuous at every $z$-point of the latter description, at which $B(z)=0$.

The nonhomogeneous problem (4.19) has a solution (except at z, c-points)

$$
\Phi(x)=\psi(x) \Phi_{0}(x),
$$

where $\psi(x)$ is from (4.18a) and $\Phi_{0}(x)$ is given by (4.21); this solution is "regular" in $C(L)$.

Consider now the special cases

$$
\psi^{+}(t)+\psi^{-}(t)=0
$$




$$
\Phi^{+}(t)+\Phi^{-}(t)=B(t)
$$

where $B(t)$ is as in (4.19). In the previous developments we put $A(t)=-1$. This function is uniform on $L^{0}$. Hence the use of uniformizing function (4.9a) is obviated; that is, one may put $u_{j}(x)=1$. We obtain a solution of (4.23) in the form

$$
\psi(x)=v(x) \gamma(x) \exp \left\{\frac{1}{2} \int_{L} \frac{d y}{y-x}\right\}
$$

where

$$
\gamma(x)=\prod_{c}(x-c)^{\kappa(c)},
$$

the product being extended over the $c$-points of $L$; here integers $\kappa(c)$ are chosen so that

$$
\left|\psi^{+^{-1}}(t)\right| \leqq e^{\beta_{1}(t)}|t-c| \nu(c)
$$

for $t$ on $L$ near $c$-points $\left(\beta_{1}(t)\right.$ bounded); in this connection we use a procedure indicated previously; moreover,

$$
v(x)=\left(x-d_{1}\right)^{s_{1}} \cdots\left(x-d_{\mu^{\prime}-1}\right)^{s_{\mu^{\prime}-1}}
$$

with $d_{\nu}$ in $O_{\nu}$; the $O_{\nu}$ are connected open regions from the decomposition $C\left(L^{0}\right)=O_{1}+\cdots+O_{\mu^{\prime}}\left(O_{\mu^{\prime}}\right.$ contains $\left.\infty\right)$; the $s_{\nu}$ are arbitrary integers. The $s_{\nu}$ can be so chosen that at $\infty, \psi(x)$ is of any preassigned integral order of $|x|$. The limit $\psi^{+*}\left(z^{\prime}\right)$ can be formed at every $z^{\prime}$-point of $L$; moreover, $\psi^{+*}\left(z^{\prime}\right) \neq 0$.

A solution of (4.23a) may be given by

$$
\text { (4.24a) } \Phi(x)=\psi(x)\left[\frac{1}{2 \pi i} \int_{L} \frac{B(y) \psi^{+}(y)^{-1} d y}{y-x}+P_{N}(x)\right] \text { (in } C(L) \text {; cf. (4.24)), }
$$

where $P_{N}(x)$ is an arbitrary polynomial of degree not greater than $N$.

To obtain the $\kappa(c)$ we note $\left(4.17 \mathrm{~b}^{\prime}\right)$ and observe that, for a $c$-point of $L$, $\kappa(c)$ is the greatest integer such that

$$
\kappa(c) \leqq \pm 1 / 2,
$$

where the upper sign is used when direction along $L$ is away from $c$ and the lower sign in the contrary case. Designate points $c$ of the first kind by a-of the latter kind by $b$. Accordingly

$$
\kappa(a)=0, \quad \kappa(b)=-1, \quad \nu(c)=1 / 2
$$

and the function $\gamma(x)$ in (4.24) is seen to be of the form

$$
\gamma(x)=\prod_{b}(x-b)^{-1} \text {. }
$$

5. An inversion problem and equation (1.1). We now consider the problem 


$$
2 \Phi(t)=\frac{1}{\pi i} \int_{L} \frac{\phi(y) d y}{y-t}=f(t)
$$

where $f(t) \subset H_{p}$ on $L$ (z,c-points included).

Let us examine the consequences of existence of a solution $\phi(y)$ (on $L$ except at $z, c$-points), which is $H^{*}$ near $c$-points, which is of a Hölder class in every closed sub arc of $L$ free of $z, c$-points and which, while possibly having irremovable discontinuities of the first kind at z-points, is of a Hölder class on every suitably small closed sub arc $(z, t)$ of each of the curves of $L$, extending to or from $z$. Incidentally, such a function will be said to be of class $H^{* *}$. The function

$$
\Phi(x)=\frac{1}{2 \pi i} \int_{L} \frac{\phi(y) d y}{y-x}
$$

will then be analytic in $C(L) ; \Phi(x)=O\left(x^{-1}\right)$ (at $\left.\infty\right)$. By (3.11a) and (3.11b), $\Phi(x)$ may possibly become infinite to an order less than unity in the neighborhood of $c$-points and logarithmically infinite at z-points; $\Phi(x)$ is bounded in the vicinity of any point $t$ of $L$ distinct from $z$ and $c$-points. Such a function will be said to be of class $K_{-1}$; if $\Phi=O\left(x^{\nu}\right)($ at $\infty)$, the class will be designated by $K_{\text {, }}$.

By one of Plemelj's formulas and by (5.1)

$$
\Phi^{+}(t)+\Phi^{-}(t)=2 \Phi(t)=f(t)
$$

Let $\beta$ be the number of b-points. With (4.24a) and (4.24), (4.25a) in view, we form the function

where

$$
\Phi_{1}(x)=\psi_{1}(x) \frac{1}{2 \pi i} \int_{L} \frac{f(y) \psi_{1}^{+}(y)^{-1} d y}{y-x},
$$

$$
\psi_{1}(x)=\prod_{b}(x-b)^{-1} \exp \left(\frac{1}{2} \int_{L} \frac{d y}{y-x}\right)
$$

Clearly

$$
\Phi_{1}^{+}(t)+\Phi_{1}^{-}(t)=f(t)
$$

moreover,

$$
\Phi_{1}(x) \subset K_{-\beta-1}
$$

We put

$$
\Phi(x)=\Phi_{1}(x)+\psi_{2}(x)
$$

and note that 


$$
\psi_{2}^{+}(t)=-\overline{\psi_{2}}(t)
$$

furthermore, since $\Phi(x) \subset K_{-1}$ and by $\left(5.3^{\prime \prime}\right)$, one has

$$
\psi_{2}(x) \subset K_{-1}
$$

Now, in view of (4.23) the function $\psi_{1}(x)$, introduced previously, satisfies $\left(5.4^{\prime \prime \prime}\right)$

$$
\psi_{1}^{+}(t)=-\psi_{1}^{-}(t)
$$

also $\psi_{1}(x)=K_{-\beta}$. By $\left(5.4^{\prime}\right)$ and $\left(5.4^{\prime \prime \prime}\right)$

$$
\left(\psi_{2} / \psi_{1}\right)^{+}=\left(\psi_{2} / \psi_{1}\right)^{-}
$$

Hence $\psi_{2}(x) \psi_{1}^{-1}(x)$ is analytic in the $x$-plane except, perhaps, at $\infty, z, c$-points. One has

$$
\frac{\psi_{2}(x)}{\psi_{1}(x)}=\psi_{2}(x) \prod_{b}(x-b) \exp \left(-\frac{1}{2} \int_{L} \frac{d y}{y-x}\right)
$$

(in $C(L)$ ). The product following $\psi_{2}(x)$, above, is bounded in every finite part of $C(L)$. By $\left(5.4^{\prime \prime}\right)$

$$
\psi_{2}(x) \psi_{1}^{-1}(x) \subset O\left(x^{\beta-1}\right) .
$$

At z,c-points this function, if infinite, is of order less than unity (at z-points the infinities are "logarithmic"). Hence $\psi_{2} \psi_{1}{ }^{-1}$ is analytic in the $x$-plane except perhaps at $\infty$. Hence $\psi_{2} \psi_{1}^{-1} \equiv 0 \quad(\beta=0)$, $\equiv$ constant $(\beta=1), \equiv P_{\beta-1}(\beta>1)$, where $P_{\beta-1}$ is some polynomial of degree not greater than $\beta-1$. Accordingly

$$
\psi_{2}(x)=P_{\beta-1}(x) \psi_{1}(x),
$$

where we put $P_{-1}(x)=0$.

Thus by (5.4) and the formulas preceding (5.3)

$$
\Phi(x)=\frac{1}{2 \pi i} \int_{L} \frac{\phi(y) d y}{y-x}=\psi_{1}(x) \frac{1}{2 \pi i} \int_{L} \frac{f(y) \psi_{1}^{+}(y)^{-1} d y}{y-x}+P_{\beta-1}(x) \psi_{1}(x)
$$

(in $C(L)$; cf. $\left(5.3^{\circ}\right)$ ).

This is a conclusion following the supposition that (5.1) has a solution $\phi \subset H^{* *}$, as designated subsequent to (5.1).

By $\left(2.22^{\prime}\right)$ and $\left(5.3^{\circ}\right)$

$$
\psi_{1}^{ \pm}(t)= \pm i \prod_{b}(t-b)^{-1} \exp \left(\frac{1}{2} \int_{L} \frac{d y}{y-t}\right)= \pm i \chi(t) .
$$

Thus from (5.6) we infer

$$
\Phi^{ \pm}(t)= \pm i \chi(t)\left[\frac{1}{2 \pi i} \int_{L} \frac{f(y) \psi_{1}^{+-1}(y) d y}{y-t} \pm \frac{f(t) \psi_{1}^{+}(t)^{-1}}{2}\right] \pm i P_{\beta-1}(t) \chi(t) .
$$


Now

$$
\phi(t)=\Phi^{+}(t)-\Phi^{-}(t) .
$$

Hence

$$
\phi(t)=2 i P_{\beta-1}(t) \chi(t)+\frac{1}{\pi i} \chi(t) \int_{L} \frac{f(y) d y}{\chi(y)(y-t)},
$$

where

$$
\chi(t)=\prod_{b}(t-b)^{-1} \exp \left(\frac{1}{2} \int_{L} \frac{d \tau}{\tau-t}\right) .
$$

Accordingly, it is observed that every solution in $H^{* *}$ of (5.1) can be expressed explicitly (on L, except at $z, c$-points) in terms of $f(t)$ by means of formula (5.7), where $P_{\beta-1}$ is some polynomial of degree not greater than $\beta-1$.

Conversely, the function in the second member of (5.7), with $P_{\beta-1}(t)$ denoting any polynomial of degree not greater than $\beta-1\left(P_{-1}(t)=0\right)$, will constitute a solution in $H^{* *}$ of (5.1). We are thus able to formulate the following theorem.

THEOREM 5.1. In the field of solutions in $H^{* *}$ (cf. text after (5.1)) the general solution of (5.1) is given by (5.7), (5.7a), where $P_{\beta-1}$ is an arbitrary polynomial of degree not greater than $\beta-1\left(P_{-1}=0\right)$ and $\beta$ is the number of b-points of $L$.

We are now ready to study the integral equation (1.1),

$$
\frac{1}{\pi i} \int_{L} \frac{\kappa(t, y) \phi(y) d y}{y-t}=f(t)
$$

here $f(t) \subset H_{p}$ on $L$ (z,c-points included) ; moreover, $\kappa(t, y)$ is of the type similar to that involved in the analogous problem in $(M ; 18)$; thus the following is assumed :

$(\alpha) \kappa(y, y)$ is of a Hölder class, say $H_{h}$, on $L$, including $z, c$-points and is nowhere zero on $L$.

$$
K(t, y)=\frac{\kappa(t, y)-\kappa(t, t)}{y-t}
$$

is continuous on $L$ and is of class $H_{h}$ in $t$ and in $y$ (z,c-points included).

As in $(\mathrm{M} ; 18)$, one is able to assume without any loss of generality that

$$
\kappa(y, y)=1 \text {. }
$$

We shall seek solutions of (5.8) in the field of functions of class $H^{* *}$. We reduce (5.8) to a Fredholm integral equation with the aid of the inversion formula of Theorem 5.1 ; in this the pattern of developments is similar to that in $(\mathrm{M} ; 18 \cdots)$. 
In view of $(\beta),(\gamma)$ one may put (5.8) in the form

$$
\frac{1}{\pi i} \int_{L} \frac{\phi(y) d y}{y-t}=\bar{f}(t) \equiv f(t)-\frac{1}{\pi i} \int_{L} K(t, y) \phi(y) d y .
$$

Suppose $\phi(y)$ is a solution of $\left(5.8^{\prime}\right)$ of class $H^{* *}$. Since the infinities of $\phi$, if any, are of order less than unity the integral $\int_{L}|\phi(y)||d y|$ exists; accordingly, for all $t^{\prime}, t^{\prime \prime}$ on $L$, one has

$$
\begin{aligned}
\left|\int_{L} K\left(t^{\prime}, y\right) \phi(y) d y-\int_{L} K\left(t^{\prime \prime}, y\right) \phi(y) d y\right| & \\
& \leqq c^{*}\left|t^{\prime}-t^{\prime \prime}\right|^{h} \int_{L}|\phi(y)||d y|=c^{*}\left|t^{\prime}-t^{\prime \prime}\right|^{h},
\end{aligned}
$$

where $c^{*}$ is the previously introduced generic designation of a constant. Whence

$$
\bar{f}(t) \subset H_{\vec{p}} \quad(\bar{p}=\text { least of } p, h)
$$

on $L, z, c$-points included. Now $\left(5.8^{\prime}\right)$ (with the third member for the moment ignored) is of the form (5.1). Hence the inversion formula (5.7) will hold with $f$ replaced by $\bar{f}$. Substitution of the expression for $\bar{f}(t)$ will yield

$$
\phi(t)=\int_{L} H(t, \tau) \phi(\tau) d \tau+F(t)
$$

where

$$
\begin{aligned}
F(t) & =2 i P_{\beta-1}(t) \chi(t)+\frac{1}{\pi i} \chi(t) \int_{L} \frac{f(y) d y}{\chi(y)(y-t)}, \\
H(t, \tau) & =\frac{1}{\pi^{2}} \chi(t) \int_{L} \frac{K(y, \tau) d y}{\chi(y)(y-t)} .
\end{aligned}
$$

In establishing this use is made of a justifiable change of order of integration in

$$
\int_{L}\left[\int_{L} \frac{K(y, \tau) \phi(\tau) d \tau}{\chi(y)(y-t)}\right] d y \text {. }
$$

We recall that, in view of (3.11a) and (5.7a), we have

$$
\chi(t)=O(1)(t-c)^{-1 / 2}, \quad \chi(t)^{-1}=O(1)(t-c)^{1 / 2}
$$

on $L$ near a $c$-point. By (3.9a)

$$
\left[\int_{L} \frac{d \tau}{\tau-z^{\prime}}\right]^{*}=\lim \int_{L} \frac{d \tau}{\tau-t}=\int_{L} \frac{d \tau}{\tau-z^{\prime}}-i(\Lambda+q \pi)
$$

(as $t \rightarrow z^{\prime}$ along $L$ ); accordingly, $\chi^{*}\left(z^{\prime}\right)$ can be formed for every $z^{\prime}$-point of $L$ and one has 
(5.10a) $\chi^{*}\left(z^{\prime}\right)=\exp \left(-\frac{i}{2}(\Lambda+q \pi)\right) \prod_{b}\left(z^{\prime}-b\right)^{-1} \exp \left(\frac{1}{2} \int_{L} \frac{d \tau}{\tau-z^{\prime}}\right)$.

At points of type $z_{1}$ one has, as $t \rightarrow z$ along any portion of $L$ through $z$,

(5.10b) $\lim \chi(t)=\exp \left(-\frac{i}{2}(\Lambda+q \pi)\right) \Pi(z-b)^{-1} \exp \left(\frac{1}{2} \int_{L} \frac{d \tau}{\tau-z}\right) ;$

in (5.10b), $q$ depends on the curve of approach to $z$; the second members in (5.10a), (5.10b) are distinct from $0, \infty$. The functions $\chi^{*}, \chi^{*^{-1}}$ are of a Hölder class on every closed subset of $L$, which does not contain $z_{1}, c$-points but may possibly contain $z^{\prime}$-points; in the vicinity of a $z_{1}, c$-point these functions behave as indicated in (5.10), (5.10b). We have

$$
\int_{L} \frac{f(y) d y}{\chi(y)(y-t)}=\int_{L} \frac{f(y) d y}{\chi^{*}(y)(y-t)} ;
$$

here

$$
f(y) x^{*^{-1}(y)}
$$

is of class $H^{* *}$, is of a Hölder class near and at $z^{\prime}, c$-points; moreover, in consequence of the second relation (5.10)

Accordingly by (3.11)

$$
f(c) \chi^{*^{-1}(c)}=0 .
$$

$$
\left[\int_{L} \frac{f(y) d y}{\chi(y)(y-t)}\right]^{+}=[\cdots]-\int_{L} \frac{f(y) d y}{\chi^{*}(y)(y-c)} \neq \infty .
$$

By $\S 2$ it is thus inferred that the function (5.11) is such that one may form

$$
\left[\int_{L} \frac{f(y) d y}{x^{*}(y)(y-t)}\right]^{*}
$$

moreover, this function (5.11') is of a Hölder class near and at $z^{\prime}, c$-points and is at most logarithmically infinite at points $z_{1}$.

By (5.9a) the function

$$
F^{*}(t)=2 i P_{\beta-1}(t) \chi^{*}(t)+\frac{1}{\pi i} \chi^{*}(t)\left[\int_{L} \frac{f(y) d y}{\chi^{*}(y)(y-t)}\right]^{*}
$$

can be formed; $F^{*}(t)$ is of a Hölder class on every closed subset of $L$, not containing $z_{1}, c$-points but possibly containing $z^{\prime}$-points, and

$$
F^{*}(t)=O(1)(t-c)^{-1 / 2}
$$

for $t$ (on $L$ ) near $c$; the infinities of $F^{*}(t)$ at the $z_{1}$ are at most logarithmic.

We consider now the function 


$$
\alpha(\tau, t)=\int_{L} \frac{K(y, \tau) d y}{\chi(y)(y-t)}=\int_{L} \frac{K(y, \tau) \chi^{*^{-1}}(y) d y}{y-t} .
$$

By (5.10)

$$
K(c, \tau) \chi^{*-1}(c)=0
$$

and, in view of the text subsequent to (5.10a), $K(y, \tau) \chi^{*^{-1}}(y)$ is of a Hölder class, in $y$, on $L, z^{\prime}, c$-points included, except at points $z_{1}$; near $z_{1}$ this function is of class $H^{* *} ; \alpha(\tau, t)$ is bounded (in consequence of $\left(5.13^{\prime}\right)$ ) for $t$ near $z^{\prime}, c$-points and $\alpha(\tau, t)$ is of a Hölder class, in $t, z^{\prime}, c$-points included (uniformly with respect to $\tau$ ) and $z_{1}$ excluded; for $t$ near $z_{1}, \alpha(\tau, t)$ may be infinite at most logarithmically (cf. (3.11a), as adapted to $z_{1}$ ). In view of the continuity hypotheses regarding $K(y, \tau)$ we have

$$
K\left(y, \tau^{\prime}\right)-K\left(y, \tau^{\prime \prime}\right)=\beta\left(y, \tau^{\prime}, \tau^{\prime \prime}\right)\left(\tau^{\prime}-\tau^{\prime \prime}\right)^{h}, \quad \beta=O(1)
$$

$\left(y, \tau^{\prime}, \tau^{\prime \prime}\right.$ on $L ; z, c$-points included). Hence

We note that

$$
\left|\alpha\left(\tau^{\prime}, t\right)-\alpha\left(\tau^{\prime \prime}, t\right)\right|=\left|\tau^{\prime}-\tau^{\prime \prime}\right| h\left|\int_{L} \frac{\beta\left(y, \tau^{\prime}, \tau^{\prime \prime}\right) d y}{\chi^{*}(y)(y-t)}\right| .
$$

$$
\beta\left(c, \tau^{\prime}, \tau^{\prime \prime}\right) \chi^{*-1}(c)=0 ;
$$

moreover, $\beta \chi^{*-1}(y)$ is of a Hölder class, in $y, z^{\prime}, c$-points included, except at $z_{1}$; for $y$ near $z_{1}$ this function is in $H^{* *}$. In view of $\left(5.13^{\prime \prime}\right)$ the function

$$
\left|\int_{L} \frac{\beta\left(y, \tau^{\prime}, \tau^{\prime \prime}\right) d y}{\chi^{*}(y)(y-t)}\right|
$$

is bounded for $t$ on $L$ except for $t$ near points $z_{1}$, at which it may be logarithmically infinite. Thus $\alpha(\tau, t)$ is of a Hölder class, in $\tau, z, c$-points included (uniformly with respect to $t$ for $t$ outside neighborhoods of points $z_{1}$ ).

By (5.13) and (5.9b), on writing

$$
\phi_{0}(t)=\phi(t) \chi^{-1}(t),
$$

we express (5.9) in the form

$$
\phi_{0}(t)=\int_{L} H_{0}(t, \tau) \phi_{0}(\tau) d \tau+F_{0}(t)
$$

where

$$
H_{0}(t, \tau)=\frac{1}{\pi^{2}} \alpha(\tau, t) \chi^{*}(\tau), \quad F_{0}(t)=\chi^{-1}(t) F(t) .
$$

In view of (5.10) and of the italics subsequent to $\left(5.13^{\prime}\right),\left(5.13^{\prime \prime}\right), H_{0}(t, \tau)$ is bounded as a function of $t\left(z^{\prime}, c\right.$-points included, neighborhoods of the $z_{1}$ ex- 
cluded) for $\tau$ on any closed subset of $L$ not including $c$-points (but possibly including $z$-points); for $t$ near $z_{1}, H_{0}(t, \tau)$ may be infinite, at most logarithmically; $H_{0}(t, \tau)$ is bounded as a function of $\tau$ for $\tau$ exterior to neighborhoods of $c$-points, provided $t$ is exterior to neighborhoods of $z_{1}$-points; for $\tau$ near any $c$-point

$$
H_{0}(t, \tau)=w(t, \tau)(\tau-c)^{-1 / 2},
$$

where $|w(t, \tau)|$ is $O(1)$ for $t$ exterior to neighborhoods of $z_{1}$-points; for $t$ near $z_{1}$, $w(t, \tau)$ may be logarithmically infinite; $H_{0}(t, \tau)$ is of a Hölder class in $t\left(t \neq z_{1}\right)$ and is of a Hölder class in $\tau(\tau \neq c)$ for $t$ exterior to neighborhoods of $z_{1}$-points. $F_{0}^{*}(t)$ is of a Hölder class on $L$ for $t \neq z_{1}$; near $z_{1}, F_{0}(t)$ may be logarithmically infinite.

We shall now find a continuous transformation

$$
\bar{\tau}=h(\tau),
$$

defined for $\tau$ on $L$ and having a continuous inverse

$$
\tau=q(\bar{\tau})
$$

for $\bar{\tau}$ on $\bar{L}$, where $\bar{L}$ is the transformation of $L$ achieved by (5.16), so that, on writing

$$
\begin{aligned}
& \phi_{0}(t)=\phi_{0}(q(\bar{t}))=\bar{\phi}(\bar{t}) \\
& F_{0}(t)=F_{0}(q(\bar{t}))=\bar{F}(\bar{t}),
\end{aligned}
$$

equation (5.15) is transformed into

$$
\Phi(\bar{t})=\int_{\bar{L}} \bar{H}(\bar{t}, \bar{\tau}) \bar{\phi}(\bar{\tau}) d \bar{\tau}+\bar{F}(\bar{t}),
$$

where $\bar{H}(\bar{t}, \bar{\tau}), \bar{F}(\bar{t})$ are piecewise continuous for $\bar{t}, \bar{\tau}$ on $\bar{L}$ and have infinities, if any, of logarithmic type for $\bar{t}$ on $\bar{L}$ near points $h\left(z_{1}\right) ;(5.18)$ will thus be a regular Fredholm integral equation.

Let $c_{1}, \cdots, c_{N}$ be the $c$-points of $L$; designate by $c_{j}^{\prime}\left(\neq c_{j}\right)$ a point on $L$ suitably near (to justify the developments to follow) $c_{j}$; $L_{j}^{\prime}$ is to denote the $\operatorname{arc}\left(c_{j}, c_{j}^{\prime}\right)$ of $L$. The set $L-\sum_{j} L_{j}^{\prime}$ we decompose into a finite number of nonoverlapping $\operatorname{arcs} L_{\nu}$ so that there are no $z$-points interior any $\operatorname{arc} L_{\nu}$; one has

$$
L=\sum L_{j}^{\prime}+\sum L_{\nu}
$$

We define $h(\tau)$ as follows :

$$
h(\tau)=\left\{\begin{array}{l}
c_{j}+2\left(\tau-c_{j}\right)^{1 / 2} \\
h_{\nu, 0}+h_{\nu, 1} \tau
\end{array}\right.
$$
$\left(\tau\right.$ on $\left.L_{j}^{\prime}\right)$, $\left(h_{p, 1} \neq 0 ; \tau\right.$ on $\left.L_{v}\right)$;

the $L_{v}, h_{v, 0}, h_{r, 1}$ will be chosen so that $h(\tau)$ is continuous on $L$; for the one-half 
power of $\tau-c_{j}$, above, we choose a suitable determination. The transformations of $L_{j}^{\prime}, L_{v}$, achieved by (5.16), will be designated by $\bar{L}_{j}, \bar{L}_{\nu}$, respectively. For the transformation $\bar{L}$ of $L$ we shall have, of course, the decomposition

$$
\bar{L}=\sum \bar{L}_{j}^{\prime}+\sum \bar{L}_{\nu} \text {. }
$$

We note that the c-points of $L, \bar{L}$ are identical; $\bar{L}_{j}$ will be a neighborhood (of $\bar{L}$ ) of $c_{j}$. The inverse function $\left(5.16^{\prime}\right)$ will be

$$
q(\bar{\tau})=\left\{\begin{array}{l}
c_{j}+\left(\bar{\tau}-c_{j}\right)^{2} / 4 \\
\left(\bar{\tau}-h_{v, 0}\right) h_{\nu, 1}^{-1}
\end{array}\right.
$$

We have

$$
\begin{aligned}
\left(\tau-c_{j}\right)^{-1 / 2} d \tau & =d \bar{\tau} \\
d \tau & =h_{\nu, 1}^{-1} d \bar{\tau}
\end{aligned}
$$

With the aid of the notation (5.17), (5.17') the equation (5.15) is expressible in the form

$$
\begin{aligned}
\Phi(\bar{t}) & =\int_{\bar{L}} H_{0}(q(\bar{t}), q(\tau)) \Phi(\bar{\tau}) d q(\bar{\tau})+\bar{F}(\bar{t}) \\
& =\left(\sum_{j} \int_{\bar{L}_{j}^{\prime}}+\sum_{\nu} \int_{\bar{L}_{\nu}}\right) H_{0}(q(\bar{t}), q(\bar{\tau})) \Phi(\bar{\tau}) d q(\bar{\tau})+\bar{F}(\bar{t}) \\
& =\sum_{j} \int_{\bar{L}_{j}^{\prime}} H_{0} 2^{-1}\left(\bar{\tau}-c_{j}\right) \Phi(\bar{\tau}) d \bar{\tau}+\sum_{\nu} \int_{\bar{L}_{\nu}} H_{0} h_{\nu, 1}^{-1} \bar{\phi}(\bar{\tau}) d \bar{\tau}+\bar{F}(\bar{t}) .
\end{aligned}
$$

That is, equation (5.18) is at hand with

$$
\bar{H}(\bar{t}, \bar{\tau})=2^{-1} H_{0}\left(q(\bar{t}), q\left(^{-}\right)\right)\left(\bar{\tau}-c_{j}\right)
$$

for $\bar{t}$ on $\bar{L}$ and $\bar{\tau}$ on $\bar{L}_{j}^{\prime}$, while

$$
\bar{H}(\bar{t}, \bar{\tau})=H_{0}(q(\bar{t}), q(\bar{\tau})) h_{\nu, 1}^{-1}
$$

for $\bar{t}$ on $\bar{L}$ and for $\bar{\tau}$ on $\bar{L}_{p}$.

Since by $\left(5.19^{\prime}\right)$

$$
\left(\tau-c_{j}\right)^{-1 / 2}=2\left(\bar{\tau}-c_{j}\right)^{-1}
$$

from (5.15') it is inferred that for $\bar{t}$ on $\bar{L}$ one has, for $\bar{t} \neq h\left(z_{1}\right)$,

$$
H_{0}(q(\bar{t}), q(\bar{\tau}))=O(1)\left(\bar{\tau}-c_{j}\right)^{-1}
$$

On the other hand, in view of the properties of $H_{0}$, given subsequent to $\left(5.15^{\prime \prime}\right)$, we conclude that

$$
H_{0}(q(\bar{t}), q(\bar{\tau}))=O(1) \quad\left(\bar{t} \text { on } \bar{L} ; \bar{\tau} \text { on } \bar{L}_{p} ; \bar{t} \neq h\left(z_{1}\right)\right) .
$$

Hence by virtue of $(5.20),\left(5.20^{\prime}\right)$ it is inferred that

$$
H(\bar{t}, \bar{\tau})=O(1) \quad\left(\bar{t}, \bar{\tau} \text { on } \bar{L} ; \bar{t} \neq h\left(z_{1}\right)\right) .
$$


For $\bar{t}$ near $h\left(z_{1}\right)$ there may be logarithmic infinities. Accordingly, we have secured a situation as described in connection with (5.16)-(5.18).

TheOREM 5.2. Every solution $\phi$ of the integral equation (1.1) (cf. (5.8), ( $\alpha$ ), $(\beta),(\gamma))$ which is in $H^{* *}$ satisfies a regular Fredholm equation (5.18) of the second kind, where

$$
\Phi(\bar{t})=\phi(q(\bar{t})) \chi^{-1}(q(\bar{t}))
$$

and where the function

$$
\bar{F}(\bar{t})=\chi^{-1}(q(\bar{t})) F(q(\bar{t}))
$$

involves a certain polynomial $P_{\beta-1}$ of degree not greater than $\beta-1$ (cf. (5.9a)). Conversely, if $\Phi$ is a solution of the Fredholm equation (5.18), where $P_{\beta-1}$ is any polynomial of degree not greater than $\beta-1$, the function $\phi$, deducible from (5.21), will be in $H^{* *}$ (on $L$ ) and will be a solution of the integral equation (1.1).

The converse part of this theorem is established by observing the reversible character of the steps leading to the relation (5.18).

6. Equation (1.2). As in analogous proceedings in $(M ; 34 \cdots)$ we make the study of the equation (1.2) dependent on that of the auxiliary equation

$$
\alpha(t) \phi(t)+\frac{\beta(t)}{\pi} \int_{L} \frac{\phi(y) d y}{y-t}=f(t)
$$

here

$$
\alpha^{2}+\beta^{2} \neq 0 ; \quad \alpha, \beta \subset H_{h} ; \quad f \subset H_{p}
$$

on $L$ (z, c-points included). tion

Suppose $\phi \subset H^{* *}$ (text after (5.1)) is a solution of (6.1). We form the func-

$$
\Phi(x)=\frac{1}{2 \pi i} \int_{L} \frac{\phi(y) d y}{y-x} .
$$

Applying the original Plemelj formulas one obtains

$$
\Phi^{+}(t)=A(t) \Phi^{-}(t)+B(t)
$$

for $t$ on $L(z, c$-points excluded), where

$$
A(t)=\frac{\alpha(t)-i \beta(t)}{\alpha(t)+i \beta(t)}, \quad B(t)=\frac{f(t)}{\alpha(t)+i \beta(t)}
$$

(as in $(\mathrm{M} ; 34(4))$ ). Clearly $A(t), B(t)$ are of a Hölder class and $A(t) \neq 0$ on $L$ $(z, c$-points included). Thus $\Phi(x)$ is a particular solution, analytic in $C(L)$ and vanishing at infinity, of the problem (4.19). Let ${ }_{1} \Phi(x)$ be another solution of (4.19), obtained by methods of $\$ 4$. On letting 


$$
\Phi(x)={ }_{1} \Phi(x)+{ }_{1} \psi(x),
$$

it is inferred that

$$
{ }_{1} \psi^{+}(t)=A(t){ }_{1} \psi^{-}(t) ;
$$

that is, $1 \psi(x)$ will be a particular solution of the homogeneous boundary problem (4.5). Designate by ${ }_{2} \psi(x)$ a suitable particular solution of (4.18), obtained by methods of $\S 4$. Then dividing the equations satisfied by ${ }_{1} \psi, 2 \psi$, on writing

$$
{ }_{1} \psi(x)={ }_{2} \psi(x) \rho(x),
$$

we obtain

$$
\rho^{+}(t)=\rho^{-}(t)
$$

on $L$ except possibly at $z, c$-points. We shall seek to choose these functions ${ }_{1} \Phi(x),{ }_{2} \psi(x)$, when possible, so that $\rho(x)$ is analytic in $C(L)$ and is at $\infty$ of finite order of infinity, while in the vicinity of $z, c$-points the order of infinity of $\rho$ is less than unity; in view of (6.5), $\rho(x)$ would then be a polynomial (in the total $x$-plane).

Since, by hypothesis, $\phi$ is $H^{*}$ in the vicinity of $c$-points, that is, is $O\left(|t-c|^{-\alpha}\right)(0<\alpha<1)$ it follows by (3.11a), (3.11b) that $\Phi(x)$ is in the vicinity of $c$ of order of infinity less than unity. Accordingly, on taking account of (3.11b) and of certain other developments in $\$ 3$, we conclude that $\Phi(x)$ is of order of infinity at $z, c$-points less than unity. In estimating the order of infinity at $z$-points at which there is an irremovable first kind discontinuity, we handle the situation as if we had a number of coincident $c$-points and apply (3.11a). More precisely, the infinities of $\Phi$ at $z$-points, if there are any, are logarithmic.

We choose $v_{j}(x)$, as given in (4.13), equal identically to unity. On recalling $(4.18 \mathrm{a}),(4.17 \mathrm{a}),(4.17 \mathrm{~b}),(4.16),(4.15),(4.9 \mathrm{a}),\left(4.10^{\prime}\right)$ we define ${ }_{2} \psi(x)$ as follows:

$$
{ }_{2} \psi(x)=\theta(x) \psi_{0}(x)
$$

(in $C(L)$ );

$$
\theta(x)=\gamma(x) \exp \left\{\frac{1}{2 \pi i} \int_{\Omega} \frac{\log A(y) d y}{y-x}\right\},
$$

where

$$
\gamma(x)=\prod_{c}(x-c)^{\kappa(c)}
$$

(product over $c$-points of $\Omega$ ),

with $\kappa(c)$ an integer such that

$\left(6.6 \mathrm{a}^{\prime \prime}\right) \quad-1<\alpha(c)+\kappa(c) \leqq 0, \quad \alpha(c)=R\left[\mp \frac{\log A(c)}{2 \pi i}\right]$

( - for $a$-points, + for $b$-points); 


$$
\psi_{0}(x)=G(x) \exp \left\{\frac{1}{2 \pi i} \sum_{j=1}^{\mu} \int_{L_{j}} \frac{\log A_{j}(y) d y}{y-x}\right\},
$$

where

$$
\begin{aligned}
G(x) & =G_{1}(x) \cdots G_{\mu}(x) ; \quad G_{j}(x)=u_{j}(x)^{t^{\prime}} \\
u_{j}(x) & =\left(x-{ }_{j} d_{1}\right)^{m_{j, 1}} \cdots\left(x-{ }_{j} d_{c^{\prime}}\right)^{m_{j, \ell^{\prime}}}
\end{aligned}
$$$$
\left(t^{\prime}=t^{i, \nu} ; \text { in } O_{j_{v}}\right) \text {; }
$$$$
\left(c^{\prime}=c_{j}-1\right)
$$

$\left({ }_{j} d_{\nu}\right.$ in $\left.{ }_{j} O_{v}\right)$; moreover,

$$
A_{j}(t)=A(t) u_{j}^{-1}(t) .
$$

We recall that $L=\Omega+L^{0}$ where $\Omega$ consists of open arcs (possibly intersecting) and contains no loops, while $L^{0}$ consists of closed curves; $L^{0}=L_{1}+\cdots+L_{\mu}$, where $L_{j}$ is connected and the $L_{j}$ have no points in common; $C\left(L_{j}\right)$ $={ }_{j} O_{1}+\cdots+{ }_{j} O_{c j}$, where ${ }_{j} O_{\nu}$ is open connected and the ${ }_{j} O,(\nu=1,2, \cdots)$ have no points in common;

$$
C\left(L^{0}\right)=C\left(L_{1}\right) \cdots C\left(L_{\mu}\right)=O_{1}+\cdots+O_{\mu^{\prime}},
$$

where the $O$, are open connected and the $O_{v}$ are without common points; ${ }_{j} O_{c j}, O_{\mu^{\prime}}$ contain the point infinity; $t^{i, c_{i}}=0$.

In view of the choice of the $\kappa(c)$ the infinities of $\theta(x)$ in the finite part of $C(\Omega)$ can occur only near $c$-points and are of order less than unity; moreover, $\theta^{-1}(x)$ is bounded in every finite part of. $C(\Omega)$, including neighborhoods of $c$-points; one has

$$
\left|\theta^{-1}(x)\right|=e^{-\beta^{\prime}(x)}|x-c|^{\nu(c)} \quad(0 \leqq \nu(c)<1),
$$

where $\beta^{\prime}(x)$ is bounded, near $c$-points. $\theta(t), \theta^{-i}(t)$ are $H^{* *}$ near $z_{1}$ of $\Omega$; that is, have irremovable first kind discontinuities, but belong to a Hölder class near and at $z_{1}$ along every curve of $\Omega$ extending to or from $z_{1}$.

Since the $t^{i, c_{j}}=0$ and ${ }_{j} O_{c j}$ contains $\infty$ we have

$$
G_{j}(\infty)=1, \quad G(\infty)=1 .
$$

On writing

$$
\kappa^{\prime}=\sum_{c} \kappa(c)
$$

we observe that

$$
\gamma(x)=x^{\kappa^{\prime}} \gamma^{\prime}(x)
$$

Also, in view of $(6.6 \mathrm{a}),(6.6 \mathrm{~b})$,

$$
\left.x^{-\kappa^{\prime} \theta(x)}\right|_{x=\infty}=1,
$$$$
\psi_{0}(\infty)=1
$$

Whence

$$
\left.x^{-x^{\prime}}{ }_{2} \psi(x)\right|_{x=\infty}=1
$$


We fix a point $d_{s}$ in $O_{s}\left(s=1, \cdots, \mu^{\prime}-1\right)$ and choose the ${ }_{j} d_{v}$, as indicated in $\$ 4$, coincident with the $d_{s}$. Associated with $d_{s}$ there is an integer $p_{s}$, depending on the $m_{j, v}, t^{i, v}$ only, so that $G(x)$ is in the neighborhood of $d_{s}$ of the form

$$
G(x)=g_{s}(x)\left(x-d_{s}\right)^{p_{s}} \quad\left(g_{s}\left(d_{s}\right) \neq 0\right) ;
$$

$g_{s}(x)$ is equal to a certain polynomial in $O_{s}(s=1,2, \cdots)$; the polynomials vary with $s$; in view of $(6.7 \mathrm{a})$ for $s=\mu^{\prime}$ the polynomial is identically unity and $p_{s}=0$. In $O_{s}\left(s<\mu^{\prime}\right) G(x)$ has no poles or zeros for $x \neq d_{s} ;$ in $O_{\mu^{\prime}}$ one has $G(x)=1$.

Henceforth we assume that the integers $p_{s}$ involved in (6.8) satisfy

$$
p_{s} \leqq 0 \text {. }
$$

This hypothesis is a natural one and corresponds to analogous assumptions in (V), (G); this condition depends on the "indices" of $\log A$ for the various closed curves, involved in $L^{0}$, as well as on the directions along $L^{0}$ and on the topology of $L^{0}$.

Under (6.9)

$$
G^{-1}(x)=O(1)
$$

(in $C\left(L^{0}\right)$; also in $C(L)$ ).

Hence, by (6.6b), $\psi_{0}^{-1}(x)$ is bounded in $C(L)$. In view of $\left(6.6 \mathrm{a}, \mathrm{a}^{\prime}\right),(6.7)$ and $\left(6.7 \mathrm{c}^{\prime \prime}\right)$

$$
{ }_{2} \psi^{-1}(x)=\left\{\begin{array}{lr}
O(1) & \text { (in any finite part of } C(L)), \\
O\left(x^{-\kappa^{\prime}}\right) & (\text { at } \infty) .
\end{array}\right.
$$

In (6.4) we utilize a particular solution ${ }_{1} \Phi(x)$ of (4.19). On taking consideration of (4.22), (4.21), we let

$$
{ }_{1} \Phi(x)={ }_{2} \psi(x) \Phi_{0}(x),
$$

where

$$
\Phi_{0}(x)=\frac{1}{2 \pi i} \int_{L} \frac{B(y)_{2} \psi^{+}(y)^{-1} d y}{y-x} .
$$

We observe that ${ }_{2} \psi^{+}(y)^{-1}$ is bounded on $L$ near $z$, c-points; moreover, $B(y)_{2} \psi^{+}(y)^{-1}$ is bounded on $L$, is of Hölder class on $L$ (except at $z, c$-points), has removable discontinuities at $z^{\prime}$-points not in $L^{0} \Omega$ at which $G^{+}\left(6.6 \mathrm{~b}^{\prime}\right)$ is continuous, has first kind discontinuities (generally irremovable) at other $z$-points; $B_{2} \psi^{+^{-1}}$ is continuous at $z$-points at which $B$ (that is, $f$ ) $=0$. In consequence of some of the developments of $\$ 3$ and, in particular, by (3.11a) the infinities of $\Phi_{0}(x)$ in any finite part of $C(L)$ are at $z, c$-points and are of "logarithmic type." Thus near $c$

$$
\left|\Phi_{0}(x)\right| \leqq c^{*} \log \frac{1}{|x-c|} \quad(x \text { in } C(L)) .
$$


Under (6.3), $\left|\Phi_{0}(x)\right| \leqq c^{*}|x-c|^{-\bullet}(0<\epsilon ; \epsilon$ arbitrarily small); that is, an infinity of "logarithmic type" signifies an infinity of an arbitrarily small order.

In view of the choice of the $\kappa(c)\left(6.6 a^{\prime \prime}\right),{ }_{2} \psi(x)$ has infinities within any finite part of $C(L)$ at $c$-points only; these infinities are of order less than unity. In consequence of the italics preceding and subsequent to (6.13) from (6.12), $\left(6.7 c^{\prime \prime}\right)$ it is inferred that

$$
{ }_{1} \Phi(x)=O\left(x^{k^{\prime}-1}\right)
$$

and that in any finite part of $C(L)$ the infinities of ${ }_{1} \Phi(x)$ are of order less than unity and are at $z, c$-points; at z-points the infinities, if any, are logarithmic.

By (3.11b) and some other results in $\$ 3$ from (6.2) it is inferred that $\Phi(x)=O\left(x^{-1}\right)$ at $\infty$ and is analytic in $C(L)$ and that in $C(L)$ near $c$-points the infinities are of order less than unity, while near z-points the infinities are logarithmic at most. Now by $(6.4),{ }_{1} \psi(x)=\Phi(x)-{ }_{1} \Phi(x)$; thus, by virtue of the established properties of $\Phi(x)$ and of (6.14) and of the italics following (6.14), it is deduced that ${ }_{1} \psi(x)$ is analytic in $C(L)$ and that in the finite part of $C(L)$ the infinities of $\psi(x)$ are of order less than unity and are at $z, c$-points, being logarithmic at $z$; moreover,

$$
{ }_{1} \psi(x)=O\left(x^{-1}\right)
$$$$
\text { (if } \left.\kappa^{\prime} \leqq 0\right) \text {, }
$$$$
{ }_{1} \psi(x)=O\left(x^{x^{\prime}-1}\right)
$$$$
\left(\text { if } \kappa^{\prime}>0\right)
$$

at $\infty$. This result, together with (6.11), $\left(6.4^{\prime \prime}\right)$, implies that

$$
\rho(x)={ }_{1} \psi(x)_{2} \psi^{-1}(x)=\left\{\begin{array}{l}
O\left(x^{-\alpha^{\prime}-1}\right) \\
O\left(x^{-1}\right)
\end{array}\right.
$$
(if $\left.\kappa^{\prime}>0\right)$;

$\rho(x)$ is analytic in $C(L)$. Thus, on taking account of the text subsequent to (6.5) it is concluded that

$$
\rho(x)=P_{-\kappa^{\prime}-1}(x),
$$

where $P_{-\kappa^{\prime}-1}$ is a certain polynomial of degree not greater than $-\kappa^{\prime}-1$ $\left(P_{-\kappa^{\prime}-1}=0\right.$, if $\left.-\kappa^{\prime}-1<0\right)$.

In consequence of $(6.4),\left(6.4^{\prime \prime}\right)$ and $\left(6.15^{\prime}\right)$

$$
\Phi(x)={ }_{1} \Phi(x)+{ }_{2} \psi(x) P_{-\alpha^{\prime}-1}(x)
$$

(in $C(L)$ ). Hence by (6.2)

$\left(6.16^{\prime}\right) \phi(t)=\Phi^{+}(t)-\Phi^{-}(t)={ }_{1} \Phi^{+}(t)-{ }_{1} \Phi^{-}(t)+\left({ }_{2} \psi^{+}(t)-{ }_{2} \psi^{-}(t)\right) P_{-k^{\prime}-1}(t)$

(on $L$ ).

Let

$$
C(t)=\left\{\begin{array}{lr}
A_{j}(t) & \left(\text { on } L_{j} ; j=1, \cdots, \mu^{\prime} ;(6.6 \mathrm{c})\right), \\
A(t) & (\text { on } \Omega)
\end{array}\right.
$$

except at points common to $L^{0}, \Omega$; also we shall write 


$$
\lambda(t)=\gamma(t) \exp \left\{\frac{1}{2 \pi i} \int_{L} \frac{\log C d y}{y-t}\right\} .
$$

By (6.6), (6.6a), (6.6b) one has

$$
{ }_{2} \psi^{ \pm}(t)=\lambda(t) G^{ \pm}(t) C^{ \pm 1 / 2}(t) .
$$

Hence $(6.12 a)$ will yield

$$
\Phi_{0}(x)=\frac{1}{2 \pi i} \int_{L} \frac{B(y) \lambda^{-1}(y) C^{-1 / 2}(y) d y}{G^{+}(y)(y-x)} .
$$

Also ${ }_{2} \psi^{+}(t)-{ }_{2} \psi^{-}(t)=\lambda\left(G^{+} C^{1 / 2}-G^{-} C^{-1 / 2}\right)$ and, by virtue of (6.12),

$$
\begin{aligned}
& { }_{1} \Phi^{+}(t)-{ }_{1} \Phi^{-}(t)=2^{-1} B(t)\left[1+C^{-1}(t) G^{-}(t) G^{+^{-1}}(t)\right] \\
& +\lambda\left(G^{+} C^{1 / 2}-G^{-} C^{-1 / 2}\right) \frac{1}{2 \pi i} \int_{L} \frac{B(y) \lambda^{-1}(y) C^{-1 / 2}(y) d y}{G^{+}(y)(y-t)} .
\end{aligned}
$$

Whence by $\left(6.16^{\prime}\right)$

$$
\begin{aligned}
\phi(t)= & 2^{-1} B\left[1+\frac{C^{-1} G^{-}}{G^{+}}\right]+\lambda\left(G^{+} C^{1 / 2}-G^{-} C^{-1 / 2}\right) \\
& \cdot\left[\frac{1}{2 \pi i} \int_{L} \frac{B(y) \lambda^{-1}(y) C^{-1 / 2}(y) d y}{G^{+}(y)(y-t)}+P_{-\alpha^{\prime}-1}(t)\right]
\end{aligned}
$$

(cf. (6.17'), (6.17)). That is, if the equation (6.1) has a solution $\phi \subset H^{* *}$, then it must be expressible in the form (6.18) (under hypothesis (6.9)).

Conversely, if we define $\phi$ by formula (6.18), where $P_{-k^{\prime}-1}$ is any polynomial of degree not greater than $-\kappa^{\prime}-1$, reversing the steps which led to (6.13) we arrive at (6.16) where

By (6.4), (6.14), (6.14')

$$
\Phi(x)=\frac{1}{2 \pi i} \int_{L} \frac{\phi(y) d y}{y-x} \quad(\phi \text { from (6.18)). }
$$

$$
\Phi(x)=O\left(x^{-1}\right)=O\left(x^{x^{\prime}-1}\right)+O\left(x^{-1}\right)
$$

(if $\kappa^{\prime} \leqq 0$ ) and

$$
\Phi(x)=O\left(x^{-1}\right)=O\left(x^{k^{\prime}-1}\right)+O\left(x^{k^{\prime}-1}\right)
$$

(if $\kappa^{\prime}>0$ ). In view of $\left(1^{\circ}\right),\left(2^{\circ}\right)$, consistency “in general” requires that $\kappa^{\prime} \leqq 0$. On the other hand, if $\kappa^{\prime} \leqq 0$ the converse can be established. One may now formulate the following theorem.

Theorem 6.1. Consider equation (6.1), $\left(6.1^{\prime}\right)$ and the notation $\left(6.3^{\prime}\right)$. Suppose (6.9) holds; then every solution $\phi \subset H^{* *}$ (cf. text after (5.1)) of (6.1) is expressible in the form (6.18), where $P_{-x^{\prime}-1}(t)$ is some polynomial of degree not greater than $-\kappa^{\prime}-1\left(\kappa^{\prime}\right.$ from $(6.7 \mathrm{~b}) ; P_{-\kappa^{\prime}-1}(t) \equiv 0$ if $\left.-\kappa^{\prime}-1<0\right)$. If $\kappa^{\prime} \leqq 0$, then 
the second member of (6.18), where $P_{-\alpha^{\prime}-1}(t)$ is an arbitrary polynomial of degree not greater than $-\kappa^{\prime}-1$, is a solution of (6.1) of class $H^{* *}$.

REMARK. A condition corresponding to the inequality $\kappa^{\prime} \leqq 0$ occurs in (M).

We are now ready to study the integral equation

$$
\alpha(t) \phi(t)+\frac{1}{\pi} \int_{L} \frac{\kappa(t, y) \phi(y) d y}{y-t}=f(t) ;
$$

here $\alpha(t), \beta(t)=\kappa(t, t)$, f are subject to conditions stated in connection with (6.1'); as subsequent to (5.8), we form

$$
K(t, y)=\frac{\kappa(t, y)-\kappa(t, t)}{y-t}
$$

and assume that $K(t, y)$ is continuous on $L$ and is of class $H_{h}$ in $t$ and in $y$ $(z, c$-points included); (6.19) may be rewritten in the form

$$
\alpha(t) \phi(t)+\frac{\beta(t)}{\pi} \int_{L} \frac{\phi(y) d y}{y-t}=F(t) \equiv F(t, \phi),
$$

where

$$
F(t)=f(t)-\frac{1}{\pi} \int_{L} K(t, y) \phi(y) d y .
$$

Suppose $\phi$ is a solution of (6.19) of class $H^{* *}$ and assume (6.9). Necessarily $|\phi|$ is integrable over $L$, the infinities of $\phi(t)$ on $L$ being of order less than unity. One has

$$
\left|\int_{L}\left[K\left(t^{\prime}, y\right)-K\left(t^{\prime \prime}, y\right)\right] \phi(y) d y\right| \leqq c^{*}\left|t^{\prime}-t^{\prime \prime}\right|^{h} \int_{L}|\phi(y)||d y|
$$

for $t^{\prime}, t^{\prime \prime}$ on $L$; thus $F(t)$ is of a Hölder class on $L, z, c$-points included. Accordingly (6.20) will be of the same form as (6.1), with $F(t)$ in place of $f(t)$. By the first part of Theorem 6.1 we conclude that a relation (6.18) will hold for $\phi(t)$; that is, $\phi(t)=$ second member of (6.18) with $F$ in place of $f$. In view of $\left(6.3^{\prime}\right)$

On writing

$$
B(t)=\frac{F(t)}{\alpha(t)+i \beta(t)} .
$$

$$
\begin{aligned}
& e(t)=\lambda(t)\left[G^{+}(t) C^{1 / 2}(t)-G^{-}(t) C^{-1 / 2}(t)\right], \quad \nu(t)=\frac{\lambda^{-1}(t) C^{-1 / 2}(t) G^{+}(t)^{-1}}{\alpha(t)+i \beta(t)} \\
& \sigma(t)=\frac{2^{-1} C^{-1 / 2}(t)}{\alpha(t)+i \beta(t)}\left[G^{+}(t) C^{1 / 2}(t)+G^{-}(t) C^{-1 / 2}(t)\right] G^{+}(t)^{-1}
\end{aligned}
$$


we obtain

$$
\phi(t)=F(t) \sigma(t)+e(t)\left[P_{-\alpha^{\prime}-1}(t)+\frac{1}{2 \pi i} \int_{L} F(y) \nu(y) \frac{d y}{y-t}\right] .
$$

In view of $\left(6.20^{\prime}\right), \phi$ satisfies the integral equation

$$
\phi(t)=\int_{L} K_{1}(t, \tau) \phi(\tau) d \tau+f_{1}(t)
$$

where

$$
\begin{aligned}
f_{1}(t) & =f(t) \sigma(t)+e(t)\left[P_{-k^{\prime}-1}(t)+\frac{1}{2 \pi i} \int_{L} \frac{f(y) \nu(y) d y}{y-t}\right], \\
K_{1}(t, \tau) & =-\frac{1}{\pi} K(t, \tau) \sigma(t)-\frac{e(t)}{2 \pi^{2} i} \int_{L} \frac{K(y, \tau) \nu(y)}{y-t} d y .
\end{aligned}
$$

The computation of $K_{1}(t, \tau)$ is based on the possibility of interchange of order of integration in

$$
\int_{L} \int_{L} K(y, \tau) \nu(y) \phi(\tau) \frac{1}{y-t} d \tau d y \text {; }
$$

this possibility is established without difficulty in consequence of continuity of $K(y, \tau)$, the assumed character of $\phi(\tau)$, and in view of the fact that, in consequence of $(6.21),\left(6.3^{\prime}\right),\left(6.1^{\prime}\right),\left(6.17^{\prime}\right), \nu(y)$ is bounded on $L$ (z, $c$-points included); $\nu(y)$ is generally discontinuous on $L$; however, $\nu(y)$ is of a Hölder class in any closed subset of $L$ not containing $z, c$-points. By $\left(6.17^{\prime}\right)$ the infinities (finite in number) of $\lambda(t)$ are all of order less than unity; since

$$
G^{+}(t) C^{1 / 2}(t) \mp G^{-}(t) C^{-1 / 2}(t)
$$

is bounded on $L$ ( $z, c$-points included), the same can be said of the infinities of $e(t)$ (cf. (6.21)). Since $f(y) \nu(y)$ is bounded on $L$, the infinities of

$$
\int_{L} \frac{f(y) \nu(y)}{y-t} d y
$$

are of "logarithmic type." In view of the statement with respect to (6.22") the function $\sigma(t)$ of $(6.21)$ is bounded on $L$ (z,c-points included). Thus by $\left(6.22^{\circ}\right)$ it is inferred that $f_{1}(t)$ is of a Hölder class on $L$ (z, c-points possibly excluded); the infinities of $f_{1}(t)$ are all of order less than unity and logarithmic whenever they occur at $\mathrm{z}$.

Studying the integral

$$
Q(t, \tau)=\int_{L} \frac{K(y, \tau) \nu(y) d y}{y-t},
$$

we observe that $K(y, \tau) \nu(y)$ is bounded as a function of $y$ and is of a Hölder 
class in every closed subset of $L$ not containing $z, c$-points; hence $Q(t, \tau)$, as a function of $t$, has at most logarithmic infinities, which may occur only amongst the $z, c$-points. Now

$$
K\left(y, \tau^{\prime}\right)-K\left(y, \tau^{\prime \prime}\right)=\beta\left(y, \tau^{\prime}, \tau^{\prime \prime}\right)\left(\tau^{\prime}-\tau^{\prime \prime}\right)^{h}
$$

for $\tau^{\prime}, \tau^{\prime \prime}$ on $L$; whence

$$
\left|Q\left(t, \tau^{\prime}\right)-Q\left(t, \tau^{\prime \prime}\right)\right|=\left|\tau^{\prime}-\tau^{\prime \prime}\right| h\left|\int_{L} \frac{\beta \nu(y) d y}{y-t}\right| .
$$

Since $\beta \nu(y)$ is bounded on $L$, with possible discontinuities of the first kind at $z, c$-points, the infinities of the integral, last displayed, are of logarithmic type (for $t$ on $L$ ). As a function of $\tau, Q(t, \tau) \subset H_{h}$, with Hölder's "constant" really depending on $t$ and possibly logarithmically infinite for $t$ near $z, c$-points. In view of $\left(6.22^{\prime}\right), K_{1}(t, \tau)$, considered as a function of $t$, has infinities of order less than unity for $t$ near $c$ and of logarithmic type for $t$ near $z$; as a function of $\tau, K_{1}(t, \tau) \subset H_{h}$, with Hölder's "constant" depending on $t$ and of order of infinity less than unity for $t$ near $c$-points and of logarithmic type (at most) for $t$ near $z$-points.

Let $\omega$ be the maximum of the orders of infinity of $f_{1}(t), K_{1}(t, \tau)$ (as function of $t)$; then $0 \leqq \omega<1$. Let the $\gamma_{j}$ be the points on $L$ where these infinities occur, exclusive of $z$-points; for $t$ near $z$, in view of the above, the infinities are at most logarithmic; thus the $\gamma_{j}$ are all c-points. We form the function

$$
w(t)=\prod_{j}\left(t-\gamma_{j}\right)^{\omega}
$$

and put

$$
\phi_{0}(t)=w(t) \phi(t) ;
$$

(6.22) then takes the form

$$
\phi_{0}(t)=\int_{L} K_{0}(t, \tau) \phi_{0}(\tau) \frac{d \tau}{w(\tau)}+f_{0}(t),
$$

where the functions

$$
K_{0}(t, \tau)=K_{1}(t, \tau) w(t), \quad f_{0}(t)=f_{1}(t) w(t)
$$

are bounded for $t \neq z$; for $t$ near $z$ they are possibly logarithmically infinite; $K_{0}(t, \tau) \subset H_{h}$ in $\tau$, with Hölder's constant possibly depending on $t$.

We decompose $L$ into a finite number of $L_{i}^{\prime}, L_{\nu}$,

$$
L=\sum_{j} L_{j}^{\prime}+\sum L_{\nu}
$$

so that $L_{j}^{\prime}$ represents a small neighborhood of $\gamma_{i}$, and define a transformation 


$$
\bar{\tau}=h(\tau)=\left\{\begin{array}{lr}
\gamma_{j}+\frac{1}{1-\omega}\left(\tau-\gamma_{j}\right)^{1-\omega} & \left(\tau \text { on } L_{j}^{\prime}\right), \\
h_{\nu, 0}+h_{\nu, 1} \tau & \left(h_{v, 1} \neq 0 ; \tau \text { on } L_{v}\right),
\end{array}\right.
$$

choosing the $L_{j}^{\prime}, L_{v}, h_{p, 0}, h_{p, 1}$ so that the function $h(\tau)$ is continuous on $L$. We choose $L_{\nu}$ so that no point $z$ is interior to $L_{\nu}$. Under this transformation $L$, $L_{j}^{\prime}, L_{v}$ go into $\bar{L}, \bar{L}_{j}^{\prime}, \bar{L}_{\nu}$, respectively; the $\gamma_{j}$ are invariant under (6.26). For the inverse of this transformation one has

$$
\tau=q(\bar{\tau})= \begin{cases}\gamma_{j}+(1-\omega)^{1 /(1-\omega)}\left(--\gamma_{j}\right)^{1 /(1-\omega)} & \left(\bar{\tau} \text { on } \bar{L}_{j}^{\prime}\right), \\ \left(\bar{\tau}-h_{\nu, 0}\right) h_{\nu, 1}^{-1} & \left(\bar{\tau} \text { on } \bar{L}_{v}\right) .\end{cases}
$$

The usefulness of (6.26) is due to the differential relations

We write

$$
\begin{aligned}
\left(\tau-\gamma_{j}\right)^{-\omega} d \tau & =d \bar{\tau} & & \left(\tau \text { on } L_{j}^{\prime}\right), \\
d \tau & =h_{\nu, 1}^{-1} d \bar{\tau} & & \left(\tau \text { on } L_{\nu}\right) .
\end{aligned}
$$

$$
\begin{aligned}
\phi_{0}(t) & =\phi_{0}(q(\bar{t}))=\bar{\phi}(\bar{t}), \\
f_{0}(t) & =f_{0}(q(\bar{t}))=\bar{f}(\bar{t})
\end{aligned}
$$

and observe that equation (6.25) transforms into

$$
\Phi(\bar{t})=\int_{\bar{L}} H(\bar{t}, \bar{\tau}) d \bar{\tau}+\bar{f}(\bar{t}),
$$

where $H(\bar{t}, \bar{\tau}), \bar{f}(\bar{t})$ are piecewise continuous for $\bar{t}, \bar{\tau}$ on $\bar{L}$, with infinities (among $\bar{t}$ points $\bar{t}=h(z), \bar{t}=h(c)$ ) of logarithmic type; this is a regular Fredholm integral equation.

Suppose we consider an integral equation (6.25), where $\phi_{0}(t)$ is unknown, and where $P_{-\alpha^{\prime}-1}(t)$, involved in $f_{0}(t)$ (cf. $\left(6.25^{\prime}\right),\left(6.22^{\circ}\right)$ ), is any polynomial of degree not greater than $-\kappa^{\prime}-1\left(P_{-\kappa^{\prime}-1} \equiv 0\right.$ if $\left.-\kappa^{\prime}-1<0\right)$. Suppose $\phi(t)$ is a solution of (6.25), obtained by the formula $\phi(t)=w^{-1}(t) \phi(h(t))$ (cf. $\left(6.24^{\prime}\right)$, (6.28), (6.26)), where $\Phi$ is a solution of the regular Fredholm equation (6.29). Will $\phi(t)$ constitute a solution of class $H^{* *}$ of (6.20), that is, of (6.19)? Now (6.25) is equivalent to (6.22). We reverse now the steps which led to (6.22), going back to (6.20). This retracing of steps is analogous to a procedure followed in the proof of Theorem 6.1. Hence we must introduce the consistency condition of that theorem $\left(\kappa^{\prime} \leqq 0\right)$.

TheOREM 6.2. If (6.9) holds then every solution $\phi(t) \subset H^{* *}$ of the integral equation (6.19), (6.19') satisfies the integral equation (6.22) (cf. also (6.25), (6.24)), where $P_{-\kappa^{\prime}-1}(t)$ is a certain polynomial of degree not greater than $-\kappa^{\prime}-1$; the latter equation is equivalent to a regular Fredholm integral equation of the second kind, (6.29) (in this connection observe the continuous transformation (6.26), $\left.\left(6.26^{\prime}\right)\right)$. Conversely, if in addition to (6.9) the inequality 
$\kappa^{\prime} \leqq 0$ holds, every function of the form $\phi(t)=w^{-1}(t) \Phi(h(t))(c f .(6.24))$, where $\Phi$ is a solution of the Fredholm equation in which $P_{-x^{\prime}-1}(t)$ is any polynomial of degree not greater than $-\kappa^{\prime}-1$, will be a solution $\left(\subset H^{* *}\right)$ of the original problem (6.19).

7. Equations satisfied at $z^{\prime}$-points. In this section only those points will be considered which are of the type discussed in $\$ 2$. Extension to any $z^{\prime}$-points is not difficult. Of prime importance will be the result (3.9), (3.9a). In this connection we recall that the number $\Lambda+q \pi$, involved in (3.9a), may be different for different $z^{\prime}$-points; $q$ is unique at a $z^{\prime}$-point.

Consider now the solution (5.7) of (5.1). One may now form $\phi^{*}\left(z^{\prime}\right)$; in fact, $\phi^{*}\left(z^{\prime}\right)$ will be given by (5.7), (5.7a), where the integrals involved are "starred." Moreover, since the starred integrals are of a Hölder class on $L, z^{\prime}$ included, the same is true of $\phi^{*}(t)$. Imagine this solution substituted in (5.1). One has

$$
\frac{1}{\pi i} \int_{L} \frac{\phi(y) d y}{y-t}=\frac{1}{\pi i} \int_{L} \frac{\phi^{*}(y) d y}{y-t}=f(t)
$$

on $L$ ( $z$ excluded). By (3.9a)

$$
\left[\frac{1}{\pi i} \int_{L} \frac{\phi d y}{y-z^{\prime}}\right]^{*}=\left[\frac{1}{\pi i} \int_{L} \frac{\phi^{*} d y}{y-z^{\prime}}\right]^{*}=\frac{1}{\pi i} \int_{L} \frac{\phi^{*} d y}{y-z^{\prime}}-\frac{\Lambda+q \pi}{\pi} \phi^{*}\left(z^{\prime}\right) .
$$

Thus

$$
-\frac{\Lambda+q \pi}{\pi} \dot{\phi}^{*}\left(z^{\prime}\right)+\frac{1}{\pi i} \int_{L} \frac{\phi^{*}(y) d y}{y-z^{\prime}}=f\left(z^{\prime}\right) .
$$

We turn to equation (5.8) and consider a solution $\phi(t)$, as specified in Theorem 5.2. Now $\phi(t)$ satisfies (5.9), where by (5.9b), (5.13)

$$
H(t, \tau)=\frac{1}{\pi^{2}} \chi(t) \alpha(\tau, t) .
$$

In view of the italics subsequent to $\left(5.13^{\prime}\right)$ and subsequent to $(5.10 \mathrm{~b}), \alpha(\tau, t)$ is bounded for $t$ near $z^{\prime}, c ; \alpha(\tau, t)$ is of a Hölder class in $t\left(z^{\prime}, c\right.$ included), uniformly with respect to $\tau$; for $t$ near $z_{1}, \alpha(\tau, t)$ is logarithmically infinite; moreover, $\chi^{*}(t)$ can be formed. $\chi^{*}(t)$ is of a Hölder class excluding $z_{1}, c$ (including $z^{\prime}$ ) and has infinities of one-half order at the $c$-points; at $z_{1}, \chi^{*}(t)$ is discontinuous, but finite. Hence $H^{*}(t, \tau)$ (starring with respect to $t$ ) is of a Hölder class in $t$ ( $z^{\prime}$ included; $z_{1}, c$ excluded), uniformly with respect to $\tau$; since $|\phi(\tau)|$ is in tegrable over $L$ one obtains

$$
\left|\int_{L}\left[H(t, \tau)-H^{*}\left(z^{\prime}, \tau\right)\right] \phi(\tau) d \tau\right| \leqq c^{*}\left|t-z^{\prime}\right|^{\lambda} \int_{L}|\phi(\tau)||d \tau|=c^{*}\left|t-z^{\prime}\right|^{\lambda}
$$


Hence the function

$$
\left[\int_{L} H(t, \tau) \phi(\tau) d \tau\right]^{*}=\int_{L} H^{*}(t, \tau) \phi(\tau) d \tau
$$

can be formed; it is of a Hölder class on $L, z^{\prime}$ included ( $z_{1}, c$ possibly excluded). In view of the statement in connection with (5.12), (5.12a) we conclude that $\phi^{*}(t)$ can be formed; in fact, by (5.9)

$$
\phi^{*}(t)=\int_{L} H^{*}(t, \tau) \phi(\tau) d \tau+F^{*}(t) ;
$$

it is observed that $\phi^{*}(t)$ is of a Hölder class on $L, z^{\prime}$-points included $\left(z_{1}, c\right.$-points generally excluded). With $\phi$ in (5.8) denoting the solution in question, we may rewrite (5.8) as follows:

$$
\left.\frac{1}{\pi i} \int_{L} \frac{\kappa(t, y) \phi^{*}(y) d y}{y-t}=f(t) \quad \text { (on } L ; t \neq z\right) .
$$

Application of (3.9a) to the latter relation will yield

$$
-\frac{\Lambda+q \pi}{\pi} \kappa\left(z^{\prime}, z^{\prime}\right) \phi^{*}\left(z^{\prime}\right)+\frac{1}{\pi i} \int_{L} \frac{\kappa\left(z^{\prime}, y\right) \phi^{*}(y) d y}{y-z^{\prime}}=f\left(z^{\prime}\right) .
$$

Let $z^{0}$ denote any $z^{\prime}$-point of $L$ which is not on $L^{0} \Omega$ and let $z_{0}$ denote any $z^{0}$-point in which the functions

$$
G^{+}(t), \quad G^{-}(t)
$$

are continuous. In the sequel the notation $g^{*}$ will be used when $g(t)$ is continuous, except perhaps at z,c-points, and has first kind discontinuities at z-points; then we let

$$
g^{*}(t)=g(t)
$$

$(t$ on $L ; t \neq z, \neq c)$

and

$$
g^{*}(z)=\lim g(t)
$$$$
\text { (as } t \rightarrow z \text { along } L \text { ) }
$$

at those z-points at which the discontinuities of $g(t)$ are removable; $g^{*}(t)$ is continuous on $L$ except, perhaps, at $c$-points and except at those $z$-points at which the discontinuities of $g(t)$ are not removable. The $z_{0}$-points include at least all $z^{0}$-points on $\Omega$ and all those $z^{0}$-points of $L^{0}$ which are not intersections of the constituent closed curves of $L^{0}$, that is, $z^{0}$-points which are merely corner points of individual closed curves of $L^{0}$.

A $z$-point on $L^{0}$, not on $\Omega$, will be a $z_{0}$-point if it represents the sole common point of a number of closed constituent curves of $L^{0}$ lying outside each other, the direction along each of these curves being the same. Another example of a $z_{0}$-point is presented by a $z$-point on $L^{0}$, not on $\Omega$, which is the sole 
common point of a number of closed curves of $L^{0}$, say $K_{1}, K_{2}, \cdots, K_{\sigma}\left(K_{i+1}\right.$ inside $\left.K_{j} ; j=1, \cdots, \sigma-1\right)$, the directions along these being alternate.

By (6.17), $\log C(t)$ is of a Hölder class on $L$ excluding, perhaps, $z$-points in $L^{0} \Omega$. In view of $\left(6.17^{\prime}\right)$ and (3.9a) the function $\lambda^{*}$ can be formed; one has

$$
\lambda^{*}\left(z^{0}\right)=\gamma\left(z^{0}\right) \exp \left\{\frac{1}{2 \pi i} \int_{L} \frac{\log C(y) d y}{y-z^{0}}\right\} C^{\alpha}\left(z_{0}\right) \quad\left(\alpha=-\frac{1}{2 \pi}(\Lambda+q \pi)\right) ;
$$

$\lambda^{*}(t)$ is of a Hölder class, $z^{0}$ included (other z-points generally excluded); $\lambda^{*}\left(z^{0}\right) \neq 0 ; \lambda^{*}(t)^{-1}$ has the same properties.

In consequence of $(6.21), \nu^{*}(t)$ is of a Hölder class, $z_{0}$ included (other $z$-points excluded). We write

$$
\sigma(t, \tau)=\int_{L} \frac{K(y, \tau) \nu(y) d y}{y-t}
$$

and note that the limit

$$
\sigma^{*}\left(z_{0}, \tau\right)=\left.\int_{L} \frac{K(y, \tau) \nu^{*}(y) d y}{y-t}\right|_{t=z_{0}} ^{*}
$$

can be formed (starring refers to $t) ; \sigma^{*}(t, \tau)$ is of a Hölder class in $t$ near and at $z_{0}$.

By (6.21) and the italics subsequent to (7.4), $e^{*} \cdot(t)$ is of a Hölder class, $z_{0}$ included (but not necessarily at all $z^{0}$-points); $\sigma^{*}(t)$ will have a similar property.

In view of the above, of the established properties of $\nu^{*}(t), \sigma^{*}(t, \tau)$, and since $K(t, \tau)$ is of a Hölder class in $t\left(c, z\right.$ included) we can form $K_{1}^{*}(t, \tau)$ $\left(\left(6.22^{\prime}\right)\right.$; starring with respect to $\left.t\right)$. It is observed that $K_{1}^{*}(t, \tau)$ is of a Hölder class in $t, z_{0}$-points included, uniformly with respect to $\tau$ (other $z$-points excluded); thus, in particular

$$
\left|K_{1}(t, \tau)-K_{1}^{*}\left(z_{0}, \tau\right)\right|=\left|K_{1}^{*}(t, \sigma)-K_{1}^{*}\left(z_{0}, \tau\right)\right| \leqq c^{*}\left|t-z_{0}\right|^{\lambda}
$$

$(0<\lambda<1)$ for $t$ on $L$ near $z_{0}$. Following a procedure similar to the one we have employed in establishing the above result, from $\left(6.22^{\circ}\right)$ we infer that $f_{1}^{*}(t)$ can be formed and that $f_{1}^{*}$ is of a Hölder class, $z_{0}$-points included (c-points and $z$-points, not equal to $z_{0}$, generally excluded).

In consequence of $(6.22),\left(7.4^{\prime \prime}\right)$ one has

$$
\begin{aligned}
\left|\phi(t)-\int_{L} K_{1}^{*}\left(z_{0}, \tau\right) \phi(\tau) d \tau-f_{1}(t)\right| & =\left|\int_{L}\left(K_{1}(t, \tau)-K_{1}^{*}\left(z_{0}, \tau\right)\right) \phi(\tau) d \tau\right| \\
& \leqq c^{*}\left|t-z_{0}\right|^{\lambda}\left|\int_{L} \phi(\tau) d \tau\right|=c^{*}\left|t-z_{0}\right|^{\lambda},
\end{aligned}
$$

inasmuch as $|\phi|$ is integrable. Hence on noting that $\lim f_{1}(t)=f_{1}^{*}\left(z_{0}\right)$ (as $t \rightarrow z_{0}$ along $L$ ) we infer that the limit 


$$
\lim \phi(t)=\int_{L} K_{1}^{*}\left(z_{0}, \tau\right) \phi(\tau) d \tau+f_{1}^{*}\left(z_{0}\right)
$$

(as $t \rightarrow z_{0}$ ) exists. Now $\phi(t)$ is known to be of class $H^{* *} ;(7.5)$ implies that the discontinuities of $\phi$ at the $z_{0}$-points are removable. The function $\phi^{*}(t)$ accordingly can be formed; $\phi^{*}\left(z_{0}\right)$ is equal to the second member in (7.5),

$\left(7.5^{\prime}\right) \phi^{*}\left(z_{0}\right)=\int_{L} K_{1}^{*}\left(z_{0}, \tau\right) \phi(\tau) d \tau+f_{1}^{*}\left(z_{0}\right)=\int_{L} K_{1}^{*}\left(z_{0}, \tau\right) \phi^{*}(\tau) d \tau+f_{1}^{*}\left(z_{0}\right)$.

By (6.22) and (7.5)

$$
\phi(t)-\phi^{*}\left(z_{0}\right)=\int_{L}\left(K_{1}(t, \tau)-K_{1}^{*}\left(z_{0}, \tau\right)\right) \phi^{*}(\tau) d \tau+f_{1}(t)-f_{1}^{*}\left(z_{0}\right) .
$$

Hence in view of $\left(7.4^{\prime \prime}\right)$

$$
\left|\phi^{*}(t)-\phi^{*}\left(z_{0}\right)\right| \leqq c^{*}\left|t-z_{0}\right|^{\lambda} \int_{\dot{L}}\left|\phi^{*}(\tau)\right||d \tau|+\left|f_{1}^{*}(t)-f_{1}^{*}\left(z_{0}\right)\right| .
$$

Since $f_{1}^{*}$ is of a Hölder class, $z_{0}$ included, the above implies that $\phi^{*}$ is of $a$ Hölder class, $z_{0}$ included (other z-points and c-points generally excluded).

We imagine now the above function $\phi$ in (6.19). In view of the above italics and (3.9a), on letting $t \rightarrow z_{0}$, it is inferred that

$$
\left[\alpha\left(z_{0}\right)-\frac{i}{\pi}(\Lambda+q \pi) \kappa\left(z_{0}, z_{0}\right)\right] \phi^{*}\left(z_{0}\right)+\frac{1}{\pi} \int_{L} \frac{\kappa\left(z_{0}, y\right) \phi^{*}(y) d y}{y-z_{0}}=f^{*}\left(z_{0}\right) .
$$

The developments of this section may be summarized as follows.

TheOREM 7.1. Solutions of problems (5.1), (5.8) (cf. Theorem 5.2) satisfy the modified equations (7.1), (7.2), respectively, at every $z^{\prime}$-point of $L$. On taking note of the italics subsequent to (7.2) it can be stated that solutions, in accordance with Theorem 6.2, of problem (6.19) satisfy the modified equation (7.6) at every $z_{0}$-point of $L$.

We shall conclude by presenting a new application of our extended Plemelj formulas of $\$ 2$. This will be carried out for the boundary value problems of $\$ 4$. The results so obtained could be carried over to the solutions of integral equation problems of $\$ \S 5,6$, inasmuch as the latter are largely based on $\$ 4$. We introduce the notation

$$
A_{j, \mathrm{~s}}=\frac{G^{, j}}{G^{, 8}} C^{1 / 2 \pi \Omega_{j, 8},} \quad \Omega_{j, 8}=\Omega\left(\omega_{j}\right)-\Omega\left(\omega_{\varepsilon}\right)
$$

and recall that a superscript, say $j$, preceded by a comma, indicates the limit (at a $z$-point) from within the sector $W_{j}(\xi)$. 
The boundary value problem (4.18) has the solution

$$
\psi(x)=\gamma(x) G(x) \exp \left\{\frac{1}{2 \pi i} \int_{L} \frac{\log C(y) d y}{y-x}\right\}
$$

At $z^{0}$-points $\log C(y)$ is of a Hölder class; moreover,

$$
G^{+}(t)=u(t) G^{-}(t)
$$

where $u=u_{j}$ (on $L_{j}$ ), $u=1$ (on $\Omega$ ). For the approach to $z^{0}$ from within $W_{j}(\xi)$, in view of (2.20), we obtain

$$
\psi^{\cdot i}=\gamma G^{\cdot i} \exp \left\{\frac{1}{2 \pi i} \int_{L} \frac{\log C(y) d y}{y-z^{0}}+\frac{1}{2 \pi} \Omega\left(\omega_{j}\right) \log C\left(z^{0}\right)\right\} ;
$$

a similar relation will hold for the approach from within $W_{s}(\xi)(s \neq j)$. Eliminating the integral involved in the second members we obtain

$$
\psi \cdot j\left(z^{0}\right)=A_{j, s}\left(z^{0}\right) \psi^{\cdot s}\left(z^{0}\right) .
$$

With $\psi(x)$ defined as above, consider the solution

$$
\Phi(x)=\psi(x) \Phi_{0}(x), \quad \Phi_{0}(x)=\frac{1}{2 \pi i} \int_{L} \frac{B(y) \psi^{+}(y)^{-1} d y}{y-x} \quad(\text { in } C(L))
$$

of the nonhomogeneous problem (4.19); $B(y) \psi^{+*}(y)^{-1}$ exists as a unique limit at $z_{0}$-points and is of a Hölder class at $z_{0}$. By (2.20) and (7.7)

$$
\Phi \cdot j=A_{j, \& \psi}, \otimes \Phi_{0}^{, j}=A_{j, \& \psi}, \varepsilon\left[\frac{1}{2 \pi i} \int_{L} \cdots+\frac{1}{2 \pi} \Omega\left(\omega_{j}\right) B \psi^{+*-1}\right] ;
$$

substitute here $j=s$ and from the two relations eliminate the integral involved in the last members. We obtain

$$
\Phi^{, j}=A_{j, 8} \Phi^{, 8}+\frac{1}{2 \pi} \Omega_{j, 8} A_{j, 8} B \psi^{+*-1} \psi^{, 8}
$$

also, interchanging $j, s$ and eliminating $\psi^{+*}$, we deduce

$$
\frac{\Phi^{, j}}{A_{j, \&},, 8}+\frac{A_{j, 8} \Phi^{, 8}}{\psi, j}=\left(\frac{\Phi}{\psi}\right)^{, j}+\left(\frac{\Phi}{\psi}\right)^{.8} .
$$

UNIVERSITY OF ILLINOIS,

URBANA, IlL. 